\title{
Performance Analysis of an Improved PRMA Protocol for Low Earth Orbit-Mobile Satellite Systems
}

\author{
Enrico Del Re, Senior Member, IEEE, Romano Fantacci, Senior Member, IEEE, \\ Giovanni Giambene, Member, IEEE, and Walter Sergio
}

\begin{abstract}
Future mobile communication systems will be characterized by the integration of several networks at the system level. Therefore, the satellite component and the terrestrial one will use as far as possible the same protocols. Accordingly, this paper investigates the possibility of using the packet-reservation multiple-access (PRMA) technique as a medium access control protocol for low-Earth orbit-mobile satellite systems (LEO-MSS's). A modified version of the PRMA protocol, named PRMA with hindering states (PRMA-HS's), is also proposed in order to attain better performance.
\end{abstract}

Index Terms-Multiple-access schemes, satellite systems, wireless communications.

\section{INTRODUCTION}

$\mathbf{T}$ HE MOBILE communications market is the fastest growing area within the telecommunications sector [1]. Future third-generation mobile communication systems are denoted by the European Telecommunications Standards Institute (ETSI) as Universal Mobile Telecommunications Systems (UMTS) [2]. The compatible standard of the International Telecommunications Union (ITU) is the International Mobile Telecommunications after the year 2000 (IMT-2000).

The scenario envisaged in this paper is that foreseen for the future UMTS where the terrestrial and satellite components will be integrated at the system level [3], [4]. The focus here is on the medium access control (MAC) sublayer. In particular, the packet-reservation multiple-access (PRMA) protocol [5] is considered in order to share the limited radio resource among a great number of simultaneous users.

The PRMA protocol was first proposed for terrestrial microcellular networks [5]-[7]. However, its interesting features such as high efficiency [7], efficient management of voice and data traffics for future multimedia applications [8], compatibility with the ATM standard [9], dynamic allocation of PRMA carriers to cells [10], [11], support of multirate applications [12], and a quite transparent behavior with respect to user mobility have motivated us to investigate its applicability to low-Earth orbit-mobile satellite systems (LEO-MSS's) in the light of the future UMTS.

Manuscript received October 3, 1997; revised July 6, 1998. This work was supported by the Italian Space Agency and MURST.

The authors are with the Dipartimento di Ingegneria Elettronica, Università degli Studi di Firenze, 3-50139 Firenze, Italy.

Publisher Item Identifier S 0018-9545(99)04032-3.

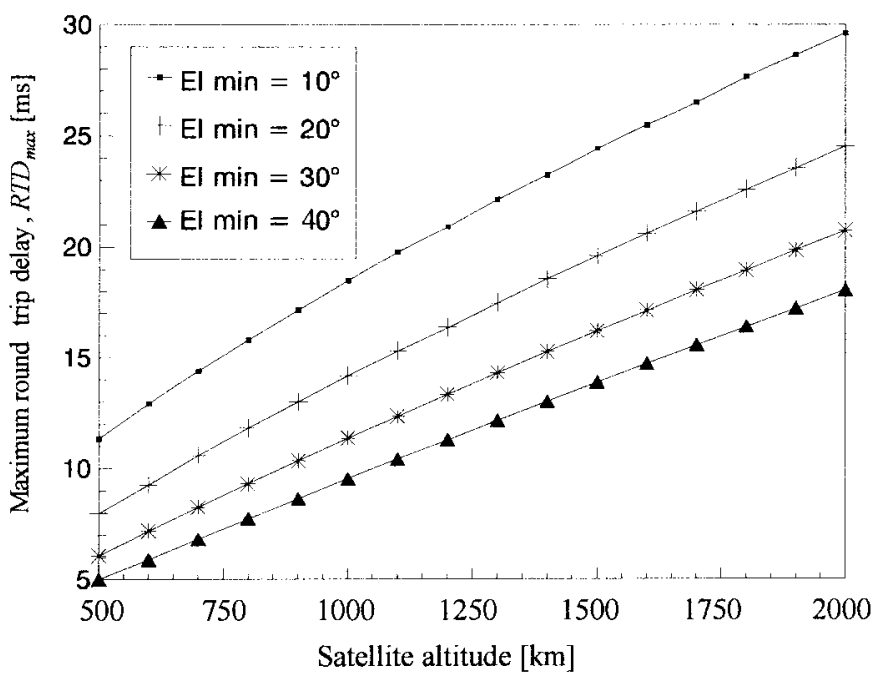

Fig. 1. Behavior of $R^{2} D_{\max }$ as a function of the LEO satellite altitude for different values of the minimum elevation angle.

LEO-MSS's offer several advantages to the satellite segment of the future UMTS. In particular, they are characterized by low-propagation delays and low-propagation attenuations which permit the use of low-power handheld terminals. Fig. 1 shows the relationship between the maximum value of the round trip delay, $\mathrm{RTD}_{\max }$, experienced by a user terminal (UT) for a given satellite altitude and a minimum elevation angle, $E l_{\text {min }}$.

Two solutions have been proposed for the implementation of an LEO-MSS able to cover the earth [13]-[15]: one is called satellite-fixed cells (adopted by the IRIDIUM system ${ }^{1}$ ), where cells are fixed with respect to satellites which move as regards the earth; the other is called earth-fixed cells (used by the TELEDESIC system ${ }^{2}$ ), where cells are fixed on the earth and satellite antenna spot beams are steered so as to point to the same area on the earth as long as possible. This paper is focused on the earth-fixed cell approach. Therefore, we have not considered aspects related to user mobility, i.e., we neglect UT cell changes during call lifetime owing to the large cell sizes obtained by satellite antennas on the earth.

\footnotetext{
${ }^{1}$ Official Web site with address: http://www.iridium.com.

${ }^{2}$ Official Web site with address: http://www.teledesic.com.
} 
However, it is important to note that the mobility management does not pose significant problems with PRMA. As soon as a UT with a call in progress in cell $x$ enters an adjacent cell $y$, a handoff procedure is started. This situation may be considered as a UT starting a talkspurt in cell $y$. The UT maintains its reservation in cell $x$ until either the signal level is acceptable, or a reservation is obtained in cell $y$ or the talkspurt ends. If an active UT leaves the coverage area of cell $x$ without a reservation in cell $y$, it will suffer from packet dropping as soon as the access delay exceeds a maximum acceptable value. However, no forced call termination is experienced by the UT. Hence, in the PRMA case the UT mobility has a slight impact on the quality of service perceived by users.

This paper is organized as follows. Section II presents an overview on the classical PRMA protocol in terrestrial cellular systems; Section III proposes the application of the PRMA protocol to MSS's. A new version of the PRMA protocol [named PRMA with hindering states (PRMA-HS's)] particularly suitable for LEO-MSS's is described in Section IV and its performance analysis is carried out in Sections $\mathrm{V}$ and VI. Finally, simulation and analysis results are compared in Section VII.

\section{Overview OF the ClassicAl PRMA PROTOCOL}

A PRMA carrier is divided into time intervals called slots with duration $T_{s}$. $N$ slots are grouped together to form a frame with duration $T_{f}$ [5]. The access to an idle slot is random, based on a permission probability $p$. Once a UT has successfully transmitted on an idle slot, it has the reservation for the exclusive use of that slot in subsequent frames. The transmission of voice is organized in packets. No more than one packet may be transmitted in $T_{s}$. Each packet contains user information bits and a header with routing, synchronization, and control information.

The PRMA protocol makes use of a speech activity detector to avoid that slots may be allocated to UT's during silent phases. When a talkspurt begins, the related UT enters the contending state: the UT tries to transmit the first packet on an available slot. In this paper, we have assumed a slow speech activity detector [7] which reveals only principal gaps within a conversation.

The reservation mechanism is made possible by a feedback channel broadcast by the controller (i.e., the base station, in the terrestrial case; whereas the satellite with on board processing capability, in MSS's) which informs all the UT's within a cell about the state of each slot of the PRMA carrier (i.e., idle/reserved).

A collision occurs in accessing the shared channel whenever two or more UT's decide to send their packets on the same slot. If we neglect the capture effect, the controller cannot recognize any UT, so it leaves the slot unreserved and all the involved UT's remain in the contending state.

Due to delay constraints in speech communications, a UT in the contending state discards the first packet from its buffer if the time to obtain a reservation exceeds a maximum value, $D_{\max }$. The value considered here for $D_{\max }$ is $32 \mathrm{~ms}$ [16].
TABLE I

SySTEM PARAMETERS

\begin{tabular}{c|c|c}
\hline Parameter & Definition & Value \\
\hline$R_{c}$ & channel bit rate & $765 \mathrm{kbit} / \mathrm{s}$ \\
\hline$R_{s}$ & speech source bit rate & $32 \mathrm{kbit} / \mathrm{s}$ \\
\hline$H_{\nu}$ & header size of a packet & $64 \mathrm{bit}$ \\
\hline$D_{\max }$ & maximum packet holding time & $32 \mathrm{~ms}$ \\
\hline
\end{tabular}

The quality of the voice transmission with PRMA is measured by the probability $P_{\text {drop }}$ that a packet is dropped from the buffer of a UT because the waiting time for transmission has exceeded $D_{\max }$. Obviously, if the number of UT's with a call in progress on the same PRMA carrier, $M$, increases, there is, on average, a greater number of UT's in the contending state, and, then, $P_{\text {drop }}$ increases. With present speech codecs, it is required $P_{\text {drop }} \leq 1 \%$ in order to cause a minimal degradation in the perceivable speech quality. Important parameters to be evaluated are the capacity of a PRMA carrier, $M_{0.01}$, defined as the maximum number of UT's with a call in progress that may share a PRMA carrier with $P_{\text {drop }} \leq 1 \%$, and the multiplexing gain $\mu_{0.01}$, which is given by the ratio between the PRMA carrier capacity $M_{0.01}$, and the equivalent capacity of a time-division multiple-access (TDMA) carrier $R_{c} / R_{s}$ (ideal case without overhead), i.e., $\mu_{0.01}=M_{0.01} R_{s} / R_{c}$ conversations/channel. Parameter $\mu_{0.01}$ is upperbounded by $\left(t_{2}+t_{1}\right) / t_{1} \approx 2.25$ conversations/channel, where $t_{1}$ is the average talkspurt duration and $t_{2}$ is the average silent duration. Parameter $\mu_{0.01}$ may reach about 1.6 conversations/channel with optimized system parameter values [7].

A further performance parameter is the throughput $\eta$, defined as the average number of packets successfully transmitted per slot. The ideal maximum value of $\eta$ is one packet/slot. For the PRMA protocol, we can usually achieve values of $\eta$ close to 0.78 packets/slot [7].

According to [7] the number of slots per frame results to be

$$
N=\left\lfloor\frac{R_{c} T_{f}}{R_{s} T_{f}+H_{v}}\right\rfloor \frac{\text { slots }}{\text { frame }}
$$

where $R_{c}, R_{s}$, and $H_{v}$ are defined in Table I and $\lfloor x\rfloor$ denotes the highest integer number less than or equal to $x$. Table I also gives the system parameter values assumed in this paper. Note that these values are different as regards [7] for reasons that will be clarified in the next section; in particular, $R_{c}$ is slightly increased.

Once the value of $T_{f}$ has been selected and $N$ has been obtained from (1), the following relationships will be used in order to obtain $T_{s}$, and the maximum reservation delay measured in slots, $D$ :

$$
T_{s}=\frac{T_{f}}{N} \quad D=\left\lceil\frac{D_{\max }}{T_{s}}\right\rceil \text { slots }
$$

where $\lceil x\rceil$ denotes the smallest integer number greater than or equal to $x$ (ceiling function). 


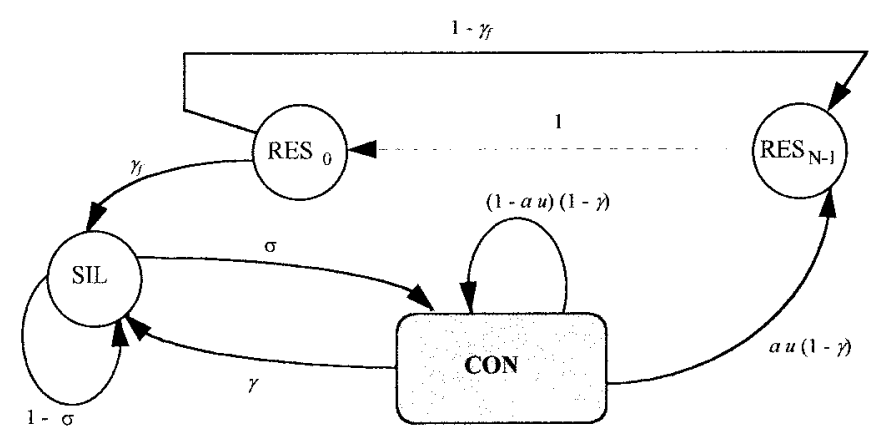

Fig. 2. The UT state diagram for the terrestrial PRMA protocol.

For instance, according to (1) and on the basis of the values shown in Table I, we obtain $N=21$ slots/frame for $T_{f}=15$ ms. Then, from (2) we have $T_{s}=0.71 \mathrm{~ms}$ and $D=46$ slots.

Fig. 2 presents the state diagram which models the UT behavior [7]. State transitions may occur at the end of each slot. A UT may be in one of the following states: silent state, $\mathrm{SIL}$, if the UT is in a silent gap; reservation states, $\mathrm{RES}_{i}$, (for $i=0, \cdots, N-1$ ), if the UT has a reservation for the next $i$ th slot; contending state, CON, if the UT is attempting to transmit on available slots. Other symbols shown in Fig. 2 are defined as: $a$ is the probability that a UT attempts to transmit on the next slot; $u$ is the probability that a transmission attempt is successful; $\sigma$ is the probability that a silent gap ends within $T_{s} ; \gamma$ is the probability that a talkspurt ends during $T_{s} ; \gamma_{f}$ is the probability that a talkspurt ends within $T_{f}$.

On the basis of the voice source model described in [7], talking and silent periods are exponentially distributed with mean values $t_{1}$ and $t_{2}$, respectively. Typical values are $1 \mathrm{~s}$ for $t_{1}$, and $1.35 \mathrm{~s}$ for $t_{2}$ [7]. According to the above assumptions, $\gamma_{f}$ is obtained as

$$
\gamma_{f}=1-(1-\gamma)^{N}
$$

In the state diagram in Fig. 2 we have also considered a transition from the CON state to the SIL one with rate $\gamma$. Such a transition takes into account that a talkspurt may end before obtaining a reservation (and, hence, it may be completely dropped). This has a high probability to occur when the CON state is congested.

\section{APPliCATION OF THE PRMA PROTOCOL TO MSS's: A FEASIBILITY STUDY}

In order to use the PRMA protocol in MSS's, we must consider the time needed to know the outcome of a reservation attempt during the contention phase (i.e., round trip delay, RTD). This time is much greater than in terrestrial cellular systems (e.g., $6 \mu \mathrm{s}$ for a terrestrial cell with 1 -km radius) and it is not negligible with respect to the slot duration (see Fig. 1). In MSS's we can consider that a UT stops contending when it is waiting for the result of a transmission attempt; this information is received from the satellite after an RTD time.

The use of the PRMA protocol in MSS's has been previously discussed by Ananasso and Delli Priscoli [17]. In particular, they showed that the high RTD values arising in geostationary MSS's (e.g., RTD is equal to $250 \mathrm{~ms}$ for a UT which sees the satellite at the zenith) prevent any application of the PRMA protocol. Conversely, the use of the PRMA protocol in MSS's based on LEO satellite constellations seems to be possible by taking into account that RTD values are in the range 5-30 $\mathrm{ms}$ (see Fig. 1). For instance, in the case RTD $=10 \mathrm{~ms}$ a contending UT has at most three attempts within $D_{\max }$ in order to transmit the first packet of its talkspurt, before dropping it.

We have considered here RTD always equal to its maximum value, $\mathrm{RTD}_{\max }$ (conservative assumption). Moreover, we have assumed $\mathrm{RTD}_{\max } \leq T_{f}-T_{s}$. Hence, when a UT makes a successful transmission attempt on an idle slot it knows the outcome of its transmission before the beginning of the same slot in the next frame. The feedback channel broadcast by the satellite provides the UT's with an updated information. If the reservation attempt has been successful, the UT can exclusively send its packets on the slot. Otherwise, the UT waits for the next idle slot, where the contention procedure is restarted.

In order to simplify the feasibility study carried out in this section, we have assumed $\mathrm{RTD}_{\max }=T_{f}-T_{s}\left(\right.$ see note $\left.{ }^{1}\right)$. Such a restriction will be removed in the next sections, where the more general case $\mathrm{RTD}_{\max } \leq T_{f}-T_{s}$ will be investigated.

According to [5]-[7], we have assumed here that a reservation packet may be erroneously received only when a collision occurs with other reservation packets.

It can be easily noted that the PRMA performance depends on both $T_{f}$ and $p$. Therefore, optimum values must be selected in order to attain the best behavior. Toward this end, we consider an MSS with system parameter values given in Table I. Note that in some practical applications, $R_{s}$ may assume a value less than that given in Table I (e.g., by means of the use of powerful codecs [18]). In these cases, the PRMA capacity is improved, i.e., higher values of $\mu_{0.01}$ are possible.

\section{A. Selection of $p$}

In deriving the optimum value of $p$, we have considered $M=30 \mathrm{UT}$ 's/carrier and two values for $T_{f}$, i.e., 5 and 15 $\mathrm{ms}$ (note that the case $T_{f}=5 \mathrm{~ms}$ by means of the assumption $T_{f} \approx \mathrm{RTD}_{\max }$, entails a limit situation for the altitude of LEO satellites ${ }^{2}$ ). From Table I and (1), it follows that we have $N=17$ slots/frame and $N=21$ slots/frame, respectively. The behavior of $P_{\text {drop }}$ as a function of $p$ for both the terrestrial and the LEO satellite cases is shown in Fig. 3. It is evident in this graph that $T_{f}=15 \mathrm{~ms}$ permits to have the lowest $P_{\text {drop }}$ on a wide range of $p$ values. This interesting result will be further discussed in Section III-B.

In the satellite case, regardless of the value of $T_{f}$ we have that if $p$ increases from 0.1 to 0.4 , the packet dropping probability decreases, i.e., the access delay is reduced. Conversely, $p$ values greater than 0.6 give rise to an increase in $P_{\text {drop }}$. Moreover, it is evident in Fig. 3 that in both the satellite cases

\footnotetext{
${ }^{1}$ Since in LEO-MSS's, RTD $\mathrm{R}_{\max } \gg T_{s}$, we can neglect $T_{s}$ with respect to $\mathrm{RTD}_{\max }$. In this case, we practically have $T_{f} \approx \mathrm{RTD}_{\max }$.

${ }^{2}$ The limit situation for LEO-MSS's with $\mathrm{RTD}_{\max }=5 \mathrm{~ms}$ has been considered only with the goal to evaluate the PRMA performance in an LEOMSS which presents the most favorable conditions for the RTD very close to a terrestrial scenario.
} 


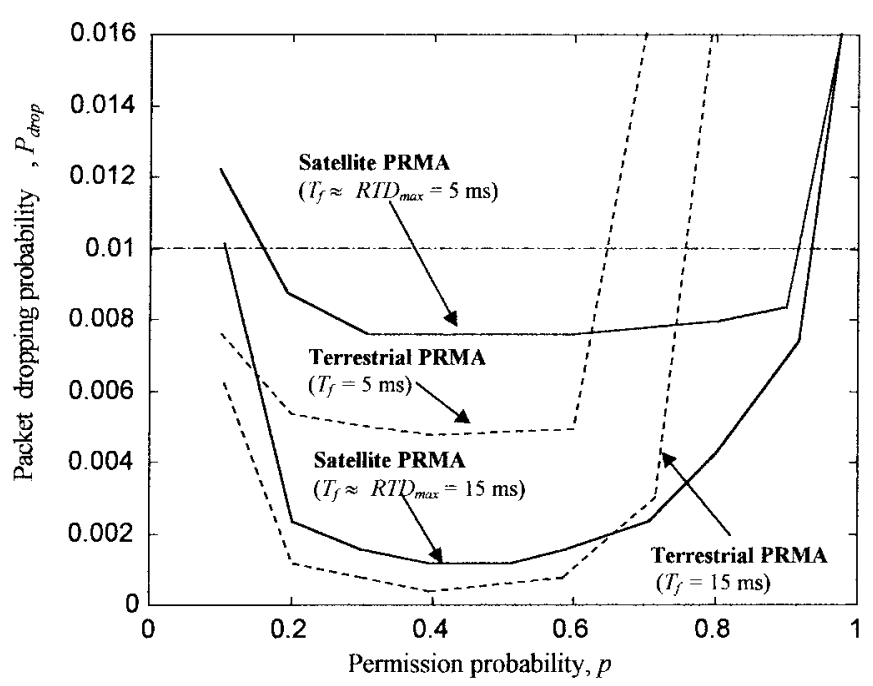

Fig. 3. Behavior of $P_{\text {drop }}$ versus $p$ with $M=30$ UT's/carrier for the PRMA protocol in both LEO satellite cases, continuous line $\left(T_{f}=\mathrm{RTD}_{\max }=5 \mathrm{~ms}\right.$ and $\left.T_{f} \approx \mathrm{RTD}_{\max }=15 \mathrm{~ms}\right)$, and terrestrial ones, dashed lines $\left(T_{f}=5 \mathrm{~ms}\right.$ and $\left.T_{f}=15 \mathrm{~ms}\right)$.

considered (i.e., $T_{f}=15$ and $5 \mathrm{~ms}$ ), $P_{\text {drop }}$ has a minimum around $p=0.4$. Therefore, $p=0.4$ can be assumed, with a good approximation, as the optimum choice for a wide range of $T_{f}$ values. This is valid in the terrestrial case as well.

\section{B. Selection of $T_{f}$}

This section discusses the impact of $T_{f}$ (equal to $\mathrm{RTD}_{\max }+$ $T_{s}$ ) on the multiplexing gain performance of the PRMA protocol. This study may help the system designer in selecting a suitable frame duration. ${ }^{3}$ Fig. 4 shows the multiplexing gain $\mu_{0.01}$ as a function of $T_{f}$ with $p=0.4$ for both the terrestrial and the satellite scenario. The system parameter values in Table I have been kept fixed. Therefore, according to (1), an increase in $T_{f}$ causes longer packets. The dependence of $\mu_{0.01}$ on $T_{f}$ highlighted in Fig. 4 can be explained as follows.

- A low value of $T_{f}$ implies to reduce the slot duration by assuming fixed values of $R_{c}$ and $R_{s}$. Therefore, a UT needs a large number of reserved slots to transmit its talkspurt. This entails a reduced number of slots available for transmission attempts by new active UT's. In this case, the multiplexing capabilities of the PRMA protocol are significantly reduced and $P_{\text {drop }}$ increases.

- An excessive value of $T_{f}$ causes a worse system performance because it leads to reduce the maximum number of attempts available for a contending UT before dropping a packet. If a frame duration greater than $40 \mathrm{~ms}$ is considered, the multiplexing gain $\mu_{0.01}$ suddenly reduces and undergoes one conversation/channel, i.e., the PRMA protocol loses its advantages as regards TDMA.

From Fig. 4, we can note a significant difference between the terrestrial case and the satellite one for high values of $T_{f}$ : in the satellite case we have a more evident reduction of $\mu_{0.01}$ when $T_{f}$ exceeds $40 \mathrm{~ms}$. This difference is due to the

\footnotetext{
${ }^{3}$ In this section, we will find a range of $T_{f}$ values that assure a good PRMA performance. Within this range, the selection of $T_{f}$ depends on both the voice codec and the packetization process.
}

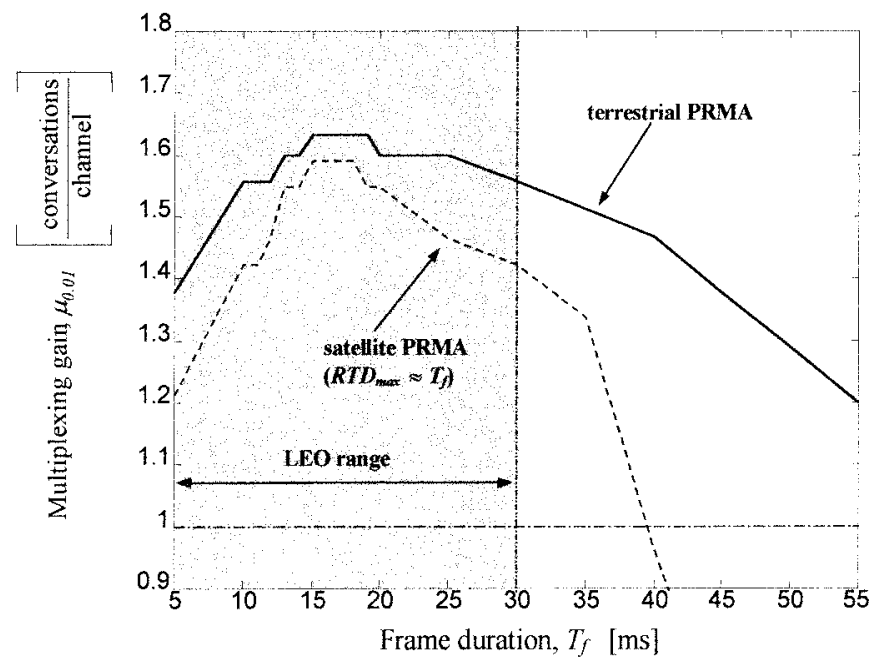

Fig. 4. The PRMA multiplexing gain as a function of $T_{f}$ with $p=0.4$ for both the terrestrial and the satellite case.

high value of RTD which significantly reduces the number of possible contention attempts within $D_{\max }$. However, in both the terrestrial case and the satellite one, $\mu_{0.01}$ has a quite flat maximum for $T_{f}$ in the range $15-16 \mathrm{~ms}^{4}$

It is important to note that, according to Fig. 4, the PRMA protocol is suitable for application in LEO systems (i.e., $\mu_{0.01}>1$ conversations/channel), but not in medium Earth orbit-MSS's (MEO-MSS's), where $\mathrm{RTD}_{\max }$ values are usually greater than $70 \mathrm{~ms}$.

We emphasize that from Figs. 3 and 4 it is evident that the same values of $p$ and $T_{f}$ (i.e., 0.4 and $15 \mathrm{~ms}$, respectively) optimize the performance of the PRMA protocol in both satellite and terrestrial cellular systems. This is a promising result in the light of the future expected integration between the terrestrial and satellite segment within UMTS.

\section{THE Proposed PRMA-HS PROTOCOL}

The main limitation for the application of the PRMA protocol to MSS's is that the high RTD values reduce the number of contention attempts within $D_{\max }$. In order to overcome this problem we propose a modified version of the classical PRMA protocol, named PRMA-HS, which allows a UT to attempt transmissions also during the time interval needed to notify the outcome of a reservation attempt (waiting time). The first successful attempt of a UT is recorded by the satellite in a data base in order to ignore any successive successful transmission attempt made by the same UT during the waiting time. ${ }^{5}$ Hence, we say that after the first successful attempt the UT enters a block of hindering states, because any successive transmission

\footnotetext{
${ }^{4}$ This result is consistent with that shown in [7], where $T_{f}=16 \mathrm{~ms}$ was selected by using slightly different system parameter values (i.e., both $p$ and the parameters of Table I).

${ }^{5}$ We refer to the voice service: a UT needs the reservation of a single slot per frame, whereas multislot reservation could be required in a multimedia system, where multirate applications are considered. The investigation of such a case is beyond the scope of this paper. However, PRMA-HS does not pose any problem for multirate applications as regards PRMA. In this case, the number of slot reservations per frame for a given UT depends on the application(s) it is running. See Section VII for more details on multimedia transmissions with PRMA-HS.
} 
attempt is useless and may only disturb the access attempts of other UT's (hindering contention). However, we will show in Section VII that despite this drawback, the PRMA-HS protocol outperforms the classical PRMA protocol.

In investigating the performance of the PRMA-HS protocol we assume $T_{f}=n \mathrm{RTD}_{\max }$, with $n$ greater than or equal to one. In the special case $n=1$, we actually consider $T_{f} \approx$ $\mathrm{RTD}_{\max }$, as already discussed. For the sake of simplicity, we will consider only integer values for $n$ which are divisors of $N$; then, $\mathrm{RTD}_{\max }$ will contain an integer number of slots. We expect that for a fixed value of $T_{f}$ the $P_{\text {drop }}$ performance can improve if $\mathrm{RTD}_{\max }$ is reduced (or equivalently $n$ increases).

On the basis of the optimization process carried out on the PRMA protocol, we have selected $T_{f}$ equal to $15 \mathrm{~ms}$ (this choice will be validated in Section VII also for PRMA-HS). Consequently, due to the possible values of $\mathrm{RTD}_{\max }$ within the LEO range, we have that reasonable values for $n$ are one-three (values of $n$ greater than three entail an $\mathrm{RTD}_{\max }$ value which is lower than that allowed by LEO systems).

In the PRMA-HS protocol a UT remains in the contending state $(\mathrm{CON})$ until it obtains a reservation. If the transmission attempt of a UT fails, it has to attempt again on the next free slot on which it has the permission to transmit. Let us assume that only one UT has attempted to transmit on a given slot; after the $\mathrm{RTD}_{\max }$ time (i.e., $N / n$ slots) the UT will know the positive outcome of its access attempt. During the waiting time, the UT continues to attempt transmissions on each idle slot according to the permission probability. ${ }^{6}$ Of course, any successful reservation attempt after the first one will be ignored by the satellite.

We can model the behavior of each UT by the Markov chain shown in Fig. 5. An active UT stays in the SIL state as long as it has no speech packet to transmit. As soon as the first packet of a talkspurt is generated the UT enters the CON state. It remains in the CON state until its attempt is successful; in this case, the UT leaves the CON state and enters the hindering states chain from $\mathrm{HIN}_{N-1}$ to $\mathrm{HIN}_{N-N / n}$; these states model the delay to know the positive outcome of a transmission attempt (i.e., $\mathrm{RTD}_{\max }=N / n$ slots). While the UT is in $\mathrm{HIN}_{i}$ states, it may continue to attempt transmissions on available slots even if it has already obtained a reservation because the positive acknowledgment is received by the UT only after the $\mathrm{RTD}_{\max }$ time. From the UT standpoint, the CON and the $\mathrm{HIN}_{i}$ states are indistinguishable. This justifies the introduction of the global contending state (GCON) in Fig. 5 . When the UT receives a positive acknowledgment, the UT leaves the hindering states, and must wait for $N-N / n-1$ slots to transmit the subsequent packet of the talkspurt or to release the access to the channel if the talkspurt is ended. In order to take into account this time, a special block of reservation states $\left(\mathrm{RES}_{i}^{\prime}\right)$, which is different from the main chain of reservation states $\left(\mathrm{RES}_{i}\right)$, has been considered. The overall time spent in the hindering states, $\mathrm{HIN}_{i}$, plus the time

\footnotetext{
${ }^{6}$ If the UT waits for a time greater than $D_{\max }$ without obtaining a reservation, it stops transmitting the first packet and starts to attempt transmissions with the second one. However, the first packet has to be considered actually dropped only if the UT does not receive a positive acknowledgment within $D_{\max }+\mathrm{RTD}_{\max }$. After the first packet is dropped, subsequent packets are discarded from the buffer after $N$ slots.
}

spent in the block of reservation states, $\mathrm{RES}_{i}^{\prime}$, must be equal to $N$ slots. Whenever a UT leaves the RES' ${ }_{i}^{\prime}$ states, with still voice packets to transmit, it enters the loop from $\operatorname{RES}_{N-1}$ to RES $_{0}$. The behavior of the UT when it is in the block of RES $i$ states is the same as for the PRMA protocol.

In the UT state diagram we have also considered the backward transition from the CON state to the SIL one with probability $\gamma$ to take into account that a talkspurt may end before obtaining a reservation.

Let $R$ denote the total number of UT's which have a reservation. Then, the expression of $a$ (i.e., the probability that a UT makes a transmission attempt on the next slot) is obtained by considering the joint probability of two independent events: 1) the next slot is unreserved (probability $1-R / N$ ) and 2) the UT obtains the permission to transmit on it (probability p). Hence

$$
a=\left(1-\frac{R}{N}\right) p
$$

Let us consider the probability $u$ that a transmission attempt has been successful and let us denote by $H$ the total number of UT's in hindering states. A UT in the CON state, which has attempted a transmission on a given slot, obtains a reservation if no other UT (in the CON state or in $\mathrm{HIN}_{i}$ states) attempts to transmit on the same slot. Therefore, probability $u$ can be expressed as follows:

$$
u= \begin{cases}(1-p)^{H}, & \text { for } C \leq 1 \text { and } \forall H \\ (1-p)^{C+H-1}, & \text { for } C>1 \text { and } \forall H\end{cases}
$$

where $C$ represents the number of UT's in the CON state.

The state vector of the PRMA-HS system is given by the following set of $2 N+2$ variables:

$$
\begin{gathered}
\Omega=\left\{S, C, R_{0}^{\prime}, R_{1}^{\prime}, \cdots, R_{N-N / n-1}^{\prime}, H_{N-N / n}, \cdots,\right. \\
\left.H_{N-1}, R_{0}, R_{1}, \cdots, R_{N-1}\right\}
\end{gathered}
$$

where $S$ is the number of UT's in the SIL state; $C$, the number of UT's in the CON state; $H_{i}$, the number of UT's waiting for a positive acknowledgment that will arrive after $i-(N-N / n)$ slots; $R_{i}^{\prime}$, the number of UT's that have a reservation on the $i$ th future slot and have left the CON state from a time less than or equal to $T_{f} ; R_{i}$, the number of UT's that hold a reservation on the $i$ th future slot and have left the CON state from a time greater than $T_{f}$.

Of course, $R_{i}, R_{i}^{\prime}$, and $H_{i}$ may be equal to either one or zero. Since a given slot can be reserved by only one UT, the following constraint must be considered:

$$
\begin{cases}R_{i}+H_{i} \leq 1, & \text { for } N-N / n-1 \leq i \leq N-1 \\ R_{i}+R_{i}^{\prime} \leq 1, & \text { for } 0 \leq i \leq N-N / n-1\end{cases}
$$

In order to obtain the total number of UT's holding a reservation, $R$, we have to sum the following terms.

- The number of UT's in hindering states $H$, since these UT's have already obtained a reservation even if they have not yet received the acknowledgment from the satellite due to RTD.

- The number of the UT's in $\mathrm{RES}_{i}^{\prime}$ states.

- The number of the UT's in $\mathrm{RES}_{i}$ states. 


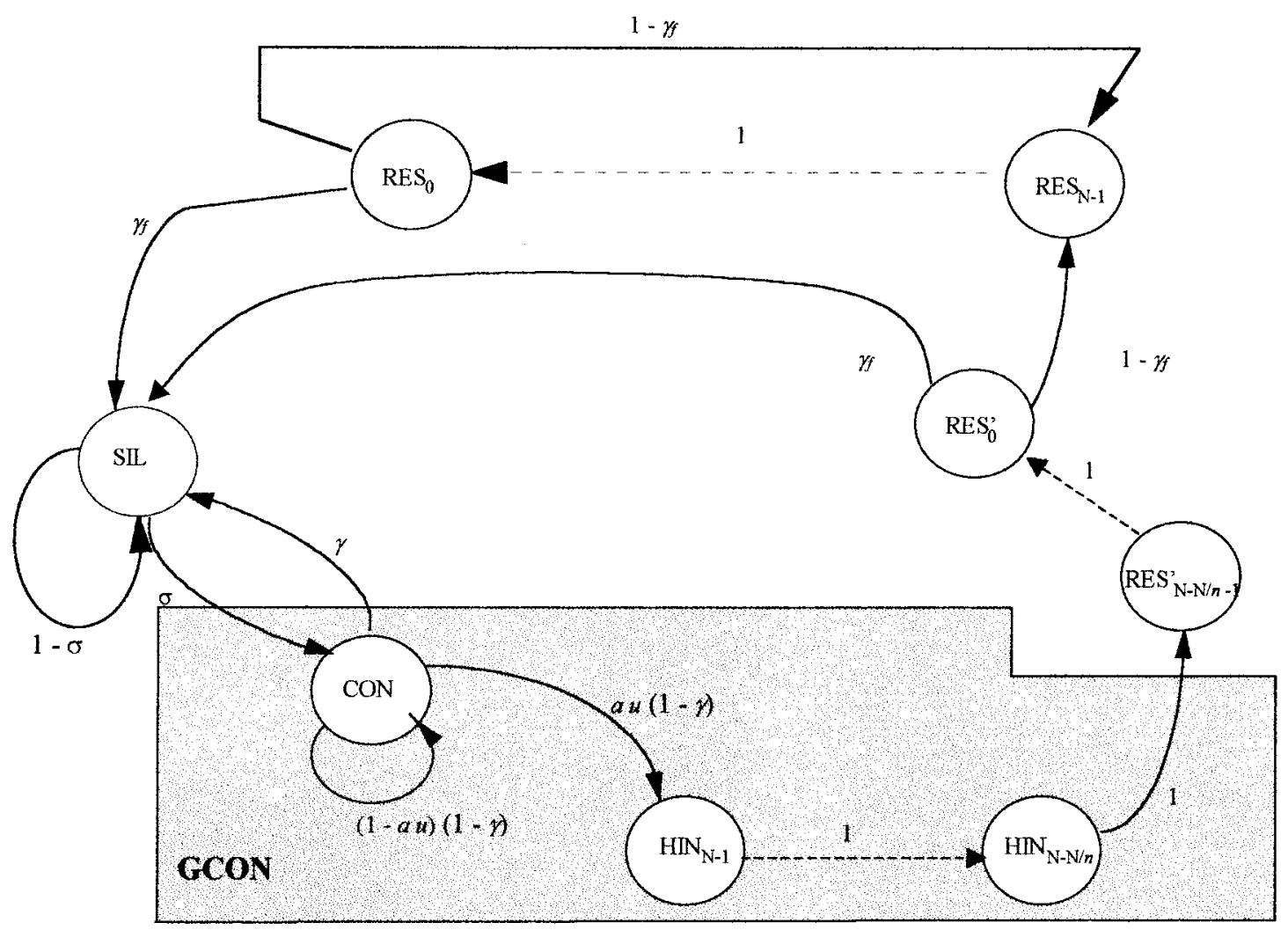

Fig. 5. The UT state diagram for the PRMA-HS technique in an LEO-MSS.

Then, according to the definitions of $R_{i}, R_{i}^{\prime}$, and $H_{i}$, we have that

$$
R=R^{*}+H
$$

where

$$
H=\sum_{i=N-N / n}^{N-1} H_{i}
$$

and

$$
R^{*}=\sum_{i=0}^{N-N / n-1} R_{i}^{\prime}+\sum_{i=0}^{N-1} R_{i} .
$$

Moreover, we must have

$$
R+S+C=M \text {. }
$$

\section{EQuilibrium PoInt AnALYsis}

The stationary probability distribution of the state vector $\Omega$ could be derived according to the standard method outlined in [19] and [20] for Markov chains. Unfortunately, that approach does not represent a viable solution in this case, since the number of possible states $\Omega$ is very high (i.e., $2^{2 N} M^{2}$ ) and the derivation of the stationary distribution can become computationally unfeasible when $N$ and $M$ have practical values. Nanda et al. [7] overcame this problem by resorting to use the equilibrium point analysis (EPA) [21], [22]. The EPA technique is based on the following definition of an equilibrium point of a system:
A point $\Omega$ in the state space is an equilibrium point if and only if it satisfies the condition that at each slot the expected change in each state variable is zero.

The advantage of the EPA approach is that it is not necessary to calculate the state transition probabilities, since it is assumed that the system is always at an equilibrium point. The EPA method reveals some problems when the system has multiple equilibrium points. A critical discussion about the accuracy of the analytical predictions obtained by the EPA method has been given in [23] and [24]. We will validate our analytical predictions by means of comparisons with simulation results.

As already discussed in Section IV, we assume that $T_{f}=n$ $\mathrm{RTD}_{\text {max }}$, where $n$ is an integer value greater or equal to one and a divisor of $N$.

The equilibrium value of each state variable is denoted by small letters, e.g., $c$ denotes the equilibrium number of UT's in the CON state. The equilibrium values of the state variables are real nonnegative numbers that can be derived by equating the inflow and the outflow for each possible state of the diagram shown in Fig. 5. From above, it is straightforward to verify that the following results hold as shown in (10)-(15), given at the bottom of the next page. Recalling that the total number of active UT's is $M$, we must have

$$
s+c+N r+N h=M .
$$

In (15), the terms $a$ and $u$ are obtained from (4) and (5) by substituting the equilibrium values to the variables $C, R$, and 
$H: c, N r+N h\left(\right.$ see note $\left.{ }^{7}\right)$ and $N h / n$, respectively,

$$
\begin{aligned}
& a=(1-r-h) p \\
& u= \begin{cases}(1-p)^{(N / n) h}, & \text { for } c \leq 1 \\
(1-p)^{c+(N / n) h-1}, & \text { for } c>1 .\end{cases}
\end{aligned}
$$

Equations (13)-(16) form a system of four equations in the four unknown equilibrium state variables $s, c, r$, and $h$. This system can be simplified in the following one, where the unknown variables are $c$ and $h$ :

$$
\begin{aligned}
F(c, h) & =\left[\frac{1}{\sigma}+\frac{N}{\gamma_{f}}\right] h+\left(1+\frac{\gamma}{\sigma}\right) c=M \\
g(c, h) & =u p c(1-\gamma)\left[1-\frac{h}{\gamma_{f}}\right]-h=0 .
\end{aligned}
$$

For each couple $(c, h)$ which fulfills this system we may obtain the corresponding values of the state variables $s$ and $r$ as

$$
\begin{aligned}
& r=\frac{1-\gamma_{f}}{\gamma_{f}} h \\
& s=\frac{[h+\gamma c]}{\sigma} .
\end{aligned}
$$

Equation (20), i.e., $g(c, h)=0$, is transcendent. Therefore, it is impossible to solve the reduced EPA system (19) and (20) by the classical substitution method; (20) represents a curve on the plane $c-h$, where the function $F(c, h)$ defined in (19) has to be evaluated. Equation (20) can be numerically solved for $c \geq 0$ and $h \geq 0$ by resorting to use the Gauss-Newton recursive method [25]. We have selected the origin $(0,0)$ as a starting point. The behavior of (20) has been shown in Fig. 6 for several values of the permission probability $p$ and for both $n=1$ and $n=3$. We can note that the curve $g(c, h)=0$ slightly depends on the value of $n$. Since parameter $n$ is present (by means of probability $u$ ) only in the second equation of the system, (19) and (20), that is, $g(c, h)=0$, we can conclude that there is a very slight dependence of the EPA solution on $n$. This interesting result will be further validated in Section VII, where it will discussed the impact of $n$ on the performance of the PRMA-HS protocol.

The EPA system (19) and (20) represents the twodimensional (2-D) transposition of the method proposed by

\footnotetext{
${ }^{7}$ The UT's that have a reservation are in $\mathrm{RES}_{i}$ states, in $\mathrm{RES}_{i}^{\prime}$ states, and in $\mathrm{HIN}_{i}$ states; all these UT's must be considered for evaluating $R$, as done in (7) and (8). We obtain the equilibrium value of $R$ by considering: $h$ UT's in each of the $N / n \mathrm{HIN}_{i}$ states, $h$ UT's in each of the $N-N / n \mathrm{RES}_{i}^{\prime}$ states, and $r$ UT's in each of the $N \mathrm{RS}_{i}$ states. Consequently, the equilibrium value of $R$ is given by $N r+N h$.
}

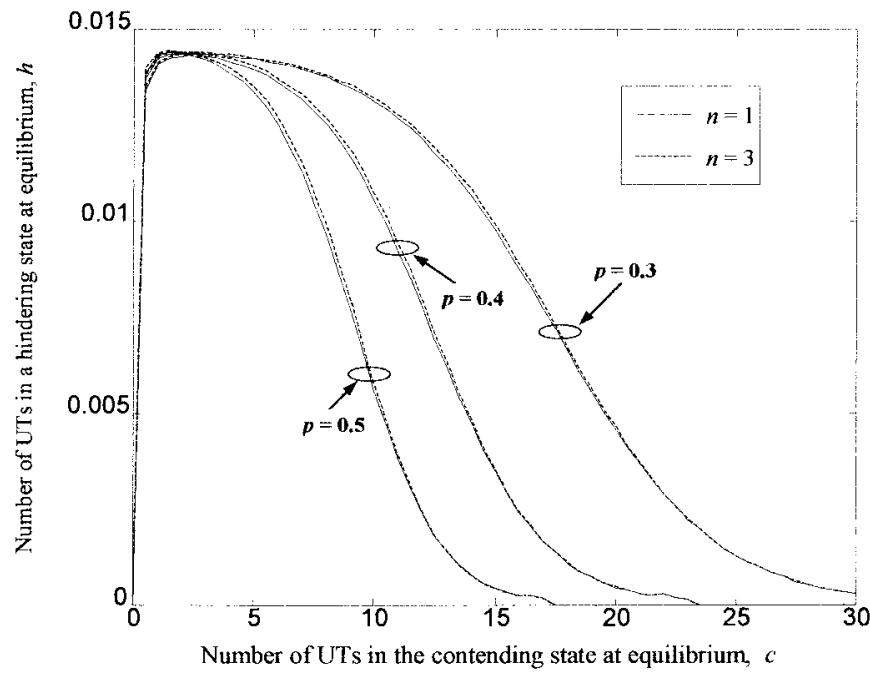

Fig. 6. Behavior of $g(c, h)=0$ in the region $c \geq 0$ and $h \geq 0$ with $N=21$ slots/frame, for several values of $p$ and $n$.

Nanda et al. in [7], where a more simple equation of the type $\mathcal{F}(c)=M$ has been solved. We are interested to find the region where the system (19) and (20) admits only one solution because this condition assures a correct application of the performance analysis (see Section VI). First, we may graphically study the solutions of the system (19) and (20) by considering the following procedure: we select a given value for $c$; according to the constraint $g(c, h)=0$, we obtain through a recursive method the corresponding value $h(c)$; then, we compute a point of the curve $F(c, h(c))$. The solutions of the system (19) and (20) may be studied on a 2-D graph as the intersections between the line at a constant height $M$ and the curve $F(c, h(c))$ as a function of $c$. This graph has been shown in Fig. 7 for $n=3, N=21$ slots/frame and several values of the probability $p$.

We note that $F(c, h(c))$ is equal to zero for $c=0$ and that $F(c, h(c))$ is greater than $M$ for $c=M$ (see note ${ }^{8}$ ). Then, the EPA system has an odd number of solutions for any selected value of $M$.

Let $F_{1}$ denote the minimum of the $F(c, h)$ curve over the constraint and let $F_{2}$ denote the maximum of $F(c, h)$ curve over the constraint. Both $F_{1}$ and $F_{2}$ depend on: $N, p, n \sigma, \gamma$.

${ }^{8}$ In (19), even if we neglect the contribution due to $h$ (generally $h \ll c$; see Fig. 6), we have that $F(c, h) \approx(1+\gamma / \sigma) c$, and this quantity is greater than $M$ for $c=M$.

$$
\begin{aligned}
& r_{0}=r_{1}=\cdots r_{N-1}=r \\
& h_{N-N / n}=h_{N-N / n-1}=\cdots h_{N-1}=h \\
& r_{0}^{\prime}=r_{1}^{\prime}=\cdots r_{N-N / n-1}^{\prime}=h \\
& \sigma s=\gamma_{f} r+\gamma_{f} h+\gamma c \\
& r=\left(1-\gamma_{f}\right) r+\left(1-\gamma_{f}\right) h \\
& \sigma s=\gamma c+\operatorname{auc}(1-\gamma)
\end{aligned}
$$

equilibrium at state:

res $_{i}$ for $i=0, \cdots, N-2$

$\mathrm{HIN}_{i}$ for $i=N-N / n, \cdots, N-1$

res $_{i}^{\prime}$ for $i=0, \cdots, N-N / n-1$

SIL

$\operatorname{res}_{N-1}$

con 


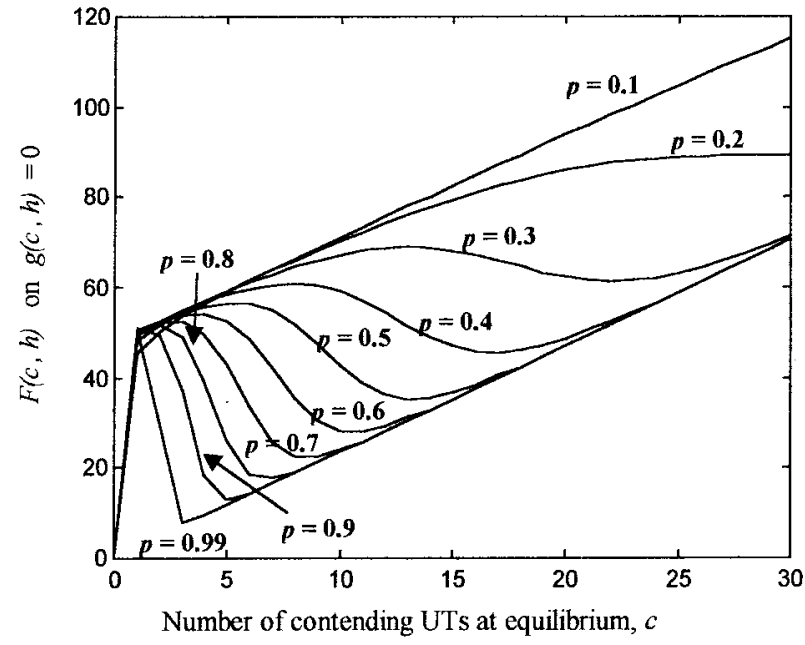

Fig. 7. Behavior of $F(c, h)$ over the restraint $g(c, h)=0$ for $N=21$ slots/frame, $n=3$ and several values of $p$.

According to the method outlined in (A.4) of the Appendix, we have obtained the behaviors shown in Fig. 8 for both $F_{1}$ and $F_{2}$ as a function of $p$ for $T_{f}=15 \mathrm{~ms}, n=3$ and the other system parameter values shown in Table I (then, $N=21$ slots/frame). For example, we have obtained: $F_{1} \approx 61.22$ and $F_{2} \approx 69.05$, for $p=0.3 ; F_{1} \approx 45.46$ and $F_{2} \approx 61.05$, for $p=0.4$. According to what is shown in the Appendix, $p_{\min }(N, n, \sigma, \gamma)$ is the minimum value of the permission probability $p$ which allows a maximum and a minimum for $F(c, h)$ over the constraint $g(c, h)=0$. Therefore, we can state that the number of solutions for the EPA system (19) and (20) is as follows.

- For $p \geq p_{\min }$ :

- one, if $0<M<F_{1}$

- three, if $F_{1} \leq M \leq F_{2}$

- one, if $M>F_{2}$.

- For $p<p_{\min }$ :

- one, for any value of $M$.

In Fig. 8, the darkest area represents a region where the values of $M$ and $p$ cause three solutions for the EPA system. In this case the system oscillates among the two extreme conditions. We must properly select the system parameter values in order to avoid this behavior. Moreover, Fig. 8 also contains a congestion curve that will be described in Section V-A, where we will prove that the PRMA-HS protocol has a low throughput (i.e., congestion) when its operating point is above this curve. Note that in Fig. 8, below the line $M=21$ UT's/carrier, the PRMA-HS protocol does not efficiently use the transmission capacity of the carrier (since we have $N=21$ slots/frame).

The gray area below the $F_{1}$ curve and below the congestion curve represents a region where the PRMA-HS performs well (there is a single EPA solution and the system is noncongested); whereas the white area above the $F_{2}$ curve and above the congestion curve is a region where the PRMA-HS protocol does not work correctly (the EPA system has a single solution, but the throughput is very low).

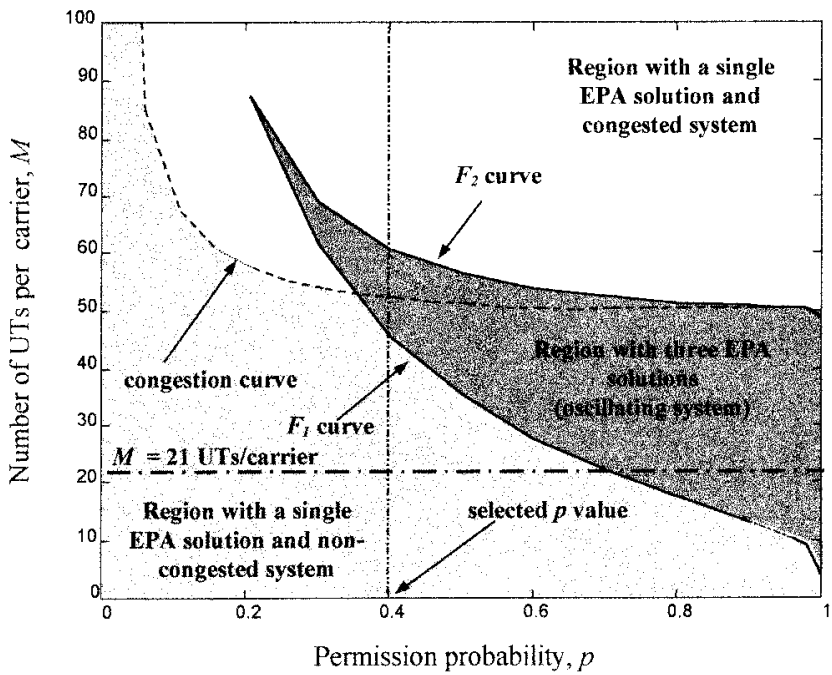

Fig. 8. Behaviors of $F_{1}$ and $F_{2}$ [respectively, the minimum and the maximum of $F(c, h)$ over $g(c, h)=0$ ] as a function of $p$, in the region $c \geq 0$ and $h \geq 0$, with $N=21$ slots/frame and $n=3$.

In conclusion, in order to have a good behavior of the PRMA-HS protocol we are interested in the region below the congestion curve and below the $F_{1}$ curve. We will show that this limitation to the value of $M$ does not reduce the usefulness of our analysis, since the fulfillment of $P_{\text {drop }} \leq 1 \%$ represents a stronger constraint.

\section{A. Stability of the Equilibrium Point}

In order to assure a good behavior of the PRMA-HS protocol it is necessary that the system is at a stable equilibrium point. In this situation, any small excursion of the state variables is forced back to their equilibrium values. Following the same approach proposed in [7], the stability of an equilibrium point can be studied by considering the outflow from the CON state, $G(c, h)$, defined as (see Fig. 5)

$$
G(c, h)=\operatorname{auc}(1-\gamma)+\gamma c
$$

where $u$ is a function of $c$ and $h$ as shown in (18).

Note that the outflow in (23) considers both the transition toward the hindering states and the transition toward the silent state that occurs when an entire talkspurt has been dropped due to system congestion.

On the basis of the EPA equations (13)-(15), we have that $G(c, h)=h+\gamma_{c}$, where $h$ is a function of $c$ according to $g(c, h)=0$. An EPA solution characterized by a given value of $c$ is a stable equilibrium point if an increase of $c$ causes an increase of the outflow from the CON state, $G(c, h)$. Therefore, a stable equilibrium point is characterized by a positive derivative of $G(c, h)$ with respect to $c$ (see the Appendix).

The expression of the outflow from the CON state, $G(c, h)$, is quite similar to $F(c, h)$; then, a similar behavior for both functions is expected. Therefore, the outflow may have both maximum and minimum points that must be investigated with the same method used in the case of $F(c, h(c))$. We have analytically studied these extreme points in the Appendix. 
From the results shown there we can conclude that both $F_{1}$ and $F_{2}$ curves in Fig. 8 permit to state (with a good approximation) the following.

- Values of $M$ and $p$ which fall outside the dark region delimited by $F_{1}$ and $F_{2}$ curves assure a single and stable solution for the EPA system. However, the system can be congested or not depending on the position with respect to the congestion curve, as discussed later in this section.

- Values of $M$ and $p$ which fall in the dark region between $F_{1}$ and $F_{2}$ curves, entail three solutions with three different $c$ values for the EPA system. The two extreme $c$ values are in the stability region; whereas the central $c$ value is unstable because it is between the $c$ values that correspond to $F_{1}$ and $F_{2}$ that are approximately the extreme points for $G(c, h(c))$. Then, the system oscillates between the two extreme $c$ values; the lowest one corresponds to a noncongested situation (i.e., high throughput), whereas the other corresponds to a congested situation (i.e., low throughput).

In Section VI-B, we will prove that when the EPA system admits a single solution, the throughput of the system, $\eta$, is proportional to $h$. Typical behaviors of $h$ as a function of $c$ are shown in Fig. 6. When $c$ increases also $h$ increases until it reaches a maximum. If $c$ increases again, $h$ and $\eta$ decrease: the system becomes congested and it cannot guarantee a good performance. Then, in order to investigate the system congestion, we need to study the point of maximum for the function $h=h(c)$ obtained from the implicit relationship $g(c, h)=0$. Let us denote by $\tilde{c}$ the point which corresponds to the maximum of the $h$ curve.

In order to formalize the definition of $\tilde{c}$, we have considered the sign of the derivative of $h$ with respect to $c$, that is given by (A.2) and (A.3) in the Appendix. We have obtained the following results.

- $d h / d c$ is always greater than zero in the region with $c$ and $h$ greater than zero and $c<1$.

- $d h / d c$ has the following behavior in the region with $c$ and $h$ greater than zero and $c \geq 1$ depending on the value of $c^{*}(p)=-1 / \ln (1-p)$ with respect to one: if $c^{*}(p)<1$, then $d h / d c$ is always negative for $c \geq 1$; otherwise, if $c^{*}(p) \geq 1$, then $d h / d c<0$ for $c>c^{*}(p), d h / d c=0$ for $c=c^{*}(p)$, and $d h / d c>0$ for $1 \leq c<c^{*}(p)$.

Note that if $p$ approaches zero, $c^{*}(p)$ tends to $+\infty$. If probability $p$ increases from zero, we have that $c^{*}(p)$ becomes more and more close to one ${ }^{+}$; the limiting condition is given by a special value for $p, p_{\text {lim }}$, which entails $c^{*}\left(p_{\text {lim }}\right)=1$. According to the definition of $c^{*}(p)$, we obtain

$$
p_{\text {lim }}=1-e^{-1} \approx 0.6321
$$

If $p>p_{\text {lim }}$, then, $c^{*}(p)<1$.

By joining together the above cases on the sign of $d h / d c$ for $c<1$ and for $c \geq 1$, we have that for $p \leq p_{\text {lim }}$ the derivative $d h / d c$ becomes equal to zero for $c=c^{*}(p)$ where we have a maximum; whereas for $p>p_{\text {lim }}$ the derivative $d h / d c$ is never equal to zero, but the $h$ curve equally presents a maximum for $c=1$, since the derivative changes its sign in this point (note that this is the connection point of two curves with slopes of different sign). Hence, we can express the maximum point for the $h$ curve as follows:

$$
\tilde{c}= \begin{cases}c^{*}(p), & \text { for } p \leq p_{\lim } \\ 1, & \text { for } p>p_{\text {lim }} .\end{cases}
$$

In conclusion, if $0<c<\tilde{c}$, the equilibrium point $c$ is in a noncongested condition; whereas if $c>\tilde{c}$, the equilibrium point $c$ is in a congested situation (case of a single EPA solution). This is similar to the behavior found in the terrestrial PRMA case [7]. The congestion curve shown in Fig. 8 (for the system parameter values given in Table I, $T_{f}=15 \mathrm{~ms}$ and $n=3)$ has been obtained as $F(\tilde{c}, h(\tilde{c}))$.

In the regions where the EPA system has a single solution (below the $F_{1}$ curve or above the $F_{2}$ curve), the analytical evaluation of the system performance can be carried out as outlined in the next section. Moreover, since it is necessary to avoid the system congestion, we are interested to the region below both the $F_{1}$ curve and the congestion curve. Hence, the values of $M$ and $p$ must be properly selected. The $M_{0.01}$ values, that will be obtained in Section VII for $p=0.4$, will be below both the congestion curve and the $F_{1}$ curve shown in Fig. 8.

\section{PeRformance AnAlysis}

The PRMA performance for voice transmissions is evaluated in terms of both the probability $P_{\text {drop }}$ and the system throughput $\eta$.

\section{A. Packet Dropping Probability}

It is straightforward to note that when a UT is in a hindering state no packet dropping can occur. Then, the discarded packets which affect $P_{\text {drop }}$ are related to a UT in the CON state shown in Fig. 5. This is a situation similar to that of the terrestrial PRMA protocol [7].

Let us consider $P_{\text {drop }}$ conditioned on having $R^{*}$ terminals which know to have a reservation, $H$ terminals in hindering states, and $C$ terminals in the contending state, i.e., $P_{\text {drop }}\left(C, H, R^{*}\right)$. We cannot consider the $R^{*}$ terminals and the $H$ terminals together, as $R$ terminals which hold a reservation because the terminals in the hindering states have a double function: they still contend even if they have already obtained a reservation.

By taking into account that $P_{\text {drop }}$ must be less than or equal to $1 \%$, we neglect the back transition from the CON state to the SIL state (see Fig. 5). We consider a tagged UT which arrives at the CON state where other $C$ terminals are already present. This UT leaves the CON state at the end of a slot (because it has obtained a reservation) if the following three independent events occur: 1) the slot is available; 2) the tagged UT has the permission to transmit on it; and 3) only the tagged UT has transmitted on this slot and then its transmission has been successful. Accordingly, the probability that the UT leaves the CON state on a slot $P_{\text {suc }}$ is given by

$$
P_{\text {suc }}\left(C, H, R^{*}\right)=\left(1-\frac{R^{*}+H}{N}\right) p(1-p)^{C+H} \text {. }
$$

Note that in (26) $P_{\text {suc }}$ is conditioned on $C, H$, and $R^{*}$. 
The tagged UT remains in the CON state at the end of a slot with probability $v\left(C, H, R^{*}\right)=1-P_{\text {suc }}\left(C, H, R^{*}\right)$. Let $t_{\mathrm{CON}}$ denote the time spent by a UT in the CON state in order to obtain a reservation. We assume that $t_{\mathrm{CON}}$ given $C, H, R^{*}$, has a geometric distribution with parameter $v=v\left(C, H, R^{*}\right)$ [7]

$$
P_{\text {con }}\left(j \mid C, H, R^{*}\right)=(1-v) v^{j-1}, \quad j=1,2, \cdots .
$$

Then, probability $P_{\text {drop }}\left(C, H, R^{*}\right)$ is computed according to [7] as

$$
P_{\text {drop }}\left(C, H, R^{*}\right)=\frac{\gamma_{f} v^{D}}{1-\left(1-\gamma_{f}\right) v^{N}} .
$$

For the special case $H+R^{*}=N$ and $M \geq N$, we have that the distribution (27) is not yet valid, since $v=1$. Under the condition that $H+R^{*}=N$, all slots are reserved and $t_{\mathrm{CON}}$ approaches infinity. Then, by taking the limit for $v \rightarrow 1$ in (28), we obtain $P_{\text {drop }}\left(C, H, R^{*}\right)=1$, for $H+R^{*}=N$.

The probability $P_{\text {drop }}$ can be computed by removing the conditioning on $C, H$, and $R^{*}$ in (28). Therefore, we need to express the joint probability distribution $\Theta_{C, H, R^{*}}\left(C, H, R^{*}\right)$ for the state variables $C, H$, and $R^{*}$. Toward this end, we resort to the Bayes rule as follows:

$$
\begin{aligned}
& \Theta_{C}, H, R^{*} \\
& \quad=\Theta_{C \mid H, R^{*}}\left(C \mid H, R^{*}\right) \Theta_{H \mid R^{*}}\left(H \mid R^{*}\right) \Theta_{R^{*}}\left(R^{*}\right)
\end{aligned}
$$

where $\Theta_{R^{*}}\left(R^{*}\right)$ is the probability that $R^{*}$ terminals are in the $\mathrm{RES}_{i}$ states, $\Theta_{H \mid R^{*}}\left(H \mid R^{*}\right)$ denotes the probability that $H$ terminals are in the $\mathrm{HIN}_{i}$ states given $R^{*}$ and $\Theta_{C \mid H, R^{*}}\left(C \mid H, R^{*}\right)$ represents the probability that $C$ terminals are in the CON state given $H$ and $R^{*}$.

In expressing $\Theta_{R^{*}}\left(R^{*}\right)$, we assume that the UT's act independently, and that each slot has the same probability to be reserved. Let $p_{\text {res }}$ be the steady probability that a slot is reserved. Since slots can be independently reserved, each with probability $p_{\text {res }}$, the following binomial distribution is considered for $\Theta_{R^{*}}\left(R^{*}\right)$ :

$$
\Theta_{R^{*}}\left(R^{*}\right)=\left(\begin{array}{l}
N \\
R^{*}
\end{array}\right) P_{\text {res }}^{R^{*}}\left(1-p_{\text {res }}\right)^{N-R^{*}}, \quad R^{*} \in[0, N]
$$

where $p_{\text {res }}$ may be obtained according to the following consideration: since we have assumed that our PRMA-HS protocol operates in the noncongested region with a single equilibrium point, the mean value of $R^{*}$ from (30) (i.e., $E\left[R^{*}\right]=N p_{\text {res }}$ ) is equal to its equilibrium value (i.e., $N r+N h-N h / n$ ); hence,

$$
p_{\text {res }}=r+\left(1-\frac{1}{n}\right) h \text {. }
$$

Note that in this study we consider that $M \geq N$ because this is the case of interest for the PRMA protocol, where we expect that more UT's share the use of the same slot.

The distribution $\Theta_{H \mid R^{*}}\left(H \mid R^{*}\right)$ has been assumed binomial, since the UT's in hindering states have an independent behavior and the maximum value of $H$ cannot exceed $N-R^{*}$ or $N / n$, whichever is less (note that the number of $\mathrm{HIN}_{i}$ states is $N / n$ and in each of them the maximum number of UT's is one; then no more than $N / n$ UT's are in $\mathrm{HIN}_{i}$ states). Hence, we have

$$
\begin{array}{r}
\Theta_{H \mid R^{*}}\left(H \mid R^{*}\right) \\
=\left(\begin{array}{c}
\min \left(N-R^{*}, N / n\right) \\
H
\end{array}\right) p_{h}^{H}\left(1-p_{h}\right)^{\min \left(N-R^{*}, N / n\right)-H}, \\
H \in\left[0, \min \left(N-R^{*}, N / n\right)\right]
\end{array}
$$

where $p_{h}$ is obtained by equating the equilibrium value of $H$ (i.e., $N h / n$ ) to its expected value which is derived according to (30)-(32); the following result is valid:

$$
p_{h}= \begin{cases}h, & \text { for } R^{*} \leq N-N / n \\ \frac{h}{n-n r-(n-1) h}, & \text { for } R^{*}>N-N / n .\end{cases}
$$

We have numerically verified that the distribution of $R=$ $R^{*}+H$ obtained by using the distributions $\Theta_{R^{*}}\left(R^{*}\right)$ and $\Theta_{H \mid R^{*}}\left(H \mid R^{*}\right)$ is very close to the binomial one used in [7].

By following the same approach proposed in [7], we model the CON state (which is shared among all the UT's) as a queuing system of the GEOM/GEOM/1 type ${ }^{9}$ [26]. Consequently, $\Theta_{C \mid H, R^{*}}\left(C \mid H, R^{*}\right)$ is geometrically distributed; this distribution must be truncated as shown in [7] because the maximum number of UT's in the CON state is upperbounded by $M-H-R^{*}$. In conclusion, $\Theta_{C \mid H, R^{*}}\left(C \mid H, R^{*}\right)$ is given by ${ }^{10}$ :

$$
\begin{aligned}
\Theta_{C \mid H, R^{*}}\left(C \mid H, R^{*}\right) & \\
= & \begin{cases}p_{0}\left(1-p_{0}\right)^{C}, & \text { for } C<M-H-R^{*} \\
\left(1-p_{0}\right)^{C}, & \text { for } C=M-H-R^{*} \\
0, & \text { otherwise }\end{cases}
\end{aligned}
$$

where $p_{0}$ is the probability that the contending state is idle. According to the EPA approach, parameter $p_{0}$ can be derived by equating the expected value of the number of UT's in the CON state, $E[C]$, to the number of contending UT's at equilibrium $c$. We obtain $E[C]$ by using the distribution of UT's in the CON state $\Theta_{C}(C)$

$$
\begin{aligned}
\Theta(C)= & \sum_{R^{*}=0}^{N} \sum_{H=0}^{\min \left(N-R^{*}, N / n\right)} \Theta_{C \mid H, R^{*}}\left(C \mid H, R^{*}\right) \\
& \cdot \Theta_{H \mid R^{*}}\left(H \mid R^{*}\right) \Theta_{R^{*}}\left(R^{*}\right), \quad C \in[0, M] .
\end{aligned}
$$

Hence, $E[C]=c$ is an equation in the unknown variable $p_{0}$ which can be numerically solved. A very good approximation of the solution is

$$
p_{0} \approx(c+1)^{-1}
$$

A validation of the expressions used for $\Theta_{R^{*}}\left(R^{*}\right)$, $\Theta_{H \mid R^{\star}}\left(H, R^{*}\right)$, and $\Theta_{C \mid H, R^{\star}}\left(C \mid H, R^{*}\right)$ has been carried

${ }^{9}$ GEOMetrically distributed interarrival times/GEOMetrically distributed service times/one server [26].

${ }^{10}$ The model of the CON state and then formula (34) are approximated because we have neglected the following aspects: 1) the arrival process of UT's to the CON state depends on the departure process of UT's from the CON state; 2) more than one UT may enter the CON state on a given slot; and 3) the time spent by a UT in the CON state depends on the number of UT's in the CON state. These approximations can be accepted if the average number of UT's in the CON state is less than one, as it is expected to obtain $P_{\text {drop }} \leq 1 \%$. 


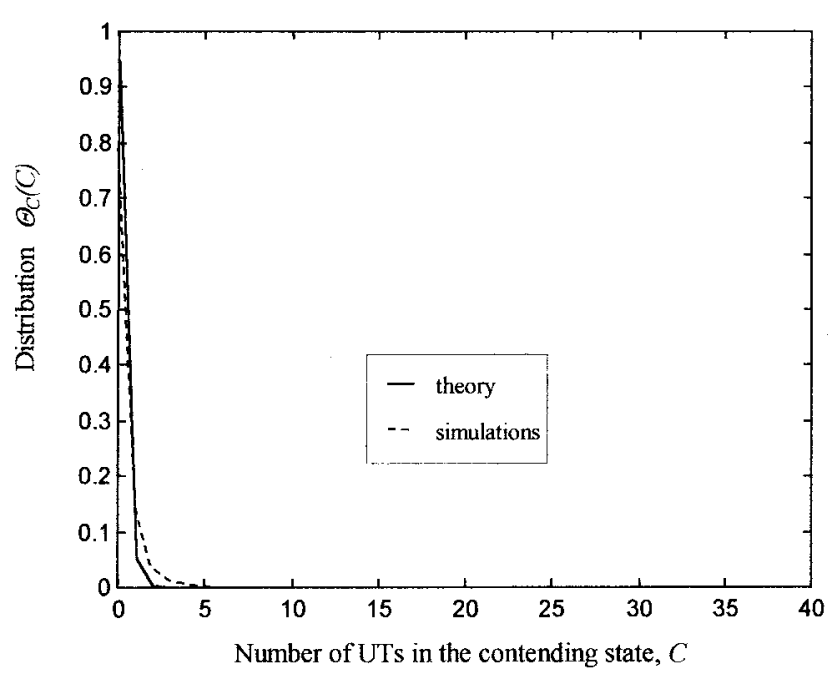

Fig. 9. Comparison between the distribution $\Theta_{C}(C)$ obtained from the analysis and the relative histogram obtained by simulations for the system parameter values shown in Table I, $T_{f}=15 \mathrm{~ms}, n=3, p=0.4$, and $M=40$ UT's/carrier.

out in Fig. 9, for $T_{f}=15 \mathrm{~ms}, p=0.4, n=3$, and $M=40$ UT's/carrier, where the distribution $\Theta_{C}(C)$ from (35) is compared to the related histogram obtained by simulations. We can note that there is an acceptable agreement between simulation results and analytical predictions. The slight differences are due to the approximations made in using (34) for $\Theta_{C \mid H, R^{*}}\left(C \mid H, R^{*}\right)$.

The performance analysis is obtained as follows: we compute the equilibrium values $c, r, s$, and $h$ according the EPA system (19) and (22); these values are used in the distributions $\Theta_{R^{*}}\left(R^{*}\right), \Theta_{H \mid R^{*}}\left(H \mid R^{*}\right)$, and $\Theta_{C \mid H, R^{*}}\left(C \mid H, R^{*}\right)$. Finally, we remove the conditioning in $P_{\text {drop }}\left(C, H, R^{*}\right)$, given by (28) with the exception $P_{\text {drop }}\left(C, H, R^{*}\right)=1$, for $R^{*}+H=$ $N$ (i.e., $v \rightarrow 1$ )

$$
\begin{aligned}
P_{\text {drop }}= & \sum_{R^{*}=0}^{N} \sum_{H=0}^{\min \left(N-R^{*}, N / n\right)} \sum_{C=0}^{M-H-R^{*}-1} P_{\text {drop }}\left(C, H, R^{*}\right) \\
& \cdot \Theta_{C \mid H, R^{*}}\left(C \mid H, R^{*}\right) \Theta_{H \mid R^{*}}\left(H \mid R^{*}\right) \Theta_{R^{*}}\left(R^{*}\right) . \text { (37) }
\end{aligned}
$$

Note that in (37) the sum on $C$ is up to $M-H-R^{*}-1$, by assuming that there is at least one UT in the SIL state that enters the CON state.

\section{B. System Throughput}

The throughput $\eta$ is the probability that a slot is reserved, $p_{r}$, by either a UT in a RES $i$ state, or a UT in a $\mathrm{HIN}_{i}$ state or a UT in a $\mathrm{RES}_{i}^{\prime}$ state. From the assumed distributions $\Theta_{R^{*}}\left(R^{*}\right)$ and $\Theta_{H \mid R^{*}}\left(H, R^{*}\right)$, we have verified that, with a very good approximation, the variable $R=R^{*}+H$ can be considered binomially distributed from zero to $N$ with parameter $p_{r}$. If we equate the expected value of $R$ (i.e., $E[R]=N p_{r}$ ) to its equilibrium value (i.e., $N r+N h$ ), we obtain

$$
\eta=p_{r}=r+h \frac{\text { packets }}{\text { slot }} .
$$

Equation (38) is only valid in the region where the EPA system admits a single solution. Moreover, on the basis of (21), we have that $\eta$ is proportional to $h$

$$
\eta=\frac{h}{\gamma_{f}} \quad \frac{\text { packets }}{\text { slot }} .
$$

For $p<p_{\min }$, the EPA system (19) and (20) admits only one solution for any value of $M$. In these conditions, $\eta$ is proportional to $h$ according to (39). For $p \geq p_{\min }$, the EPA system (19) and (20) may have either one or three solutions depending on the value of $M$. Of course, (38) and (39) can be applied only when the EPA system has only one solution. This situation can be identified as follows. Let us denote by $c_{\min }$ the EPA solution with the lowest value of $c$ for $M=F_{1}$ and by $c_{\max }$ the EPA solution with the highest value of $c$ for $M=F_{2}$. Then, for $p \geq p_{\min }$, (38) and (39) can be used only when either $c<c_{\min }$ or $c>c_{\max }$.

Let us remind that $h=h(c)$ [implicitly given by $g(c, h)=$ 0 ], has a maximum for $c=\tilde{c}$ (see Fig. 6): $\tilde{c}$ is the point where $d h / d c$ changes its sign. According to the Appendix, for $p \geq p_{\min }$ maximum and minimum points of $F(c, h(c))$ are obtained in correspondence with the intersection of $d h / d c$ with the horizontal line for $-(1+\gamma / \sigma) /\left(1 / \sigma+N / \gamma_{f}\right)<0$ : the maximum $F_{2}$ corresponds to the point $c_{F 2}$ and the minimum $F_{1}$ corresponds to the point $c_{F 1}$. Then, we have $\tilde{c}<c_{F 2}<$ $c_{F 1}<c_{\max }$.

In conclusion, for $p<p_{\min }$, the point of the maximum for $h=h(c), \tilde{c}$, always corresponds to the point of the maximum for $\eta$; whereas for $p \geq p_{\min }, \tilde{c}$, is the point of the maximum for $\eta$ only if $\tilde{c}<c_{\min }$. In Fig. 8, we have shown the congestion curve $F(\tilde{c}, h(\tilde{c}))$ for the system parameter values given in Table I, $T_{f}=15 \mathrm{~ms}$ and $n=3$. In this case, the limiting condition $\tilde{c}=c_{\min }$ is obtained for $p \approx 0.35$, i.e., when the congestion curve crosses the $F_{1}$ curve (note that, $p_{\min } \approx 0.205$, as discussed in the Appendix). Then, the point of the maximum for $h$ is also the point of the maximum of $\eta$ for $p<0.35$.

\section{Transmission Attempts by UT's in Hindering States}

When a UT obtains a reservation it will receive the positive acknowledgment after an $\mathrm{RTD}_{\max }$ time. In the meanwhile, PRMA-HS allows that other transmissions may be attempted by the same UT. These attempts may give rise to collisions with the reservation attempts of other UT's in the CON state. This is a drawback of the proposed protocol; its impact on the system performance is discussed below.

Let $P_{\text {hin }}$ be the probability that a transmission attempt made by a UT is originated from a $\mathrm{HIN}_{i}$ state and gives rise to a collision with the attempt of one UT in the CON state. Note that this is the sole case in which the transmission attempt of a UT has been both useless (because a UT in a $\mathrm{HIN}_{i}$ state has already obtained a reservation) and harmful (because it hinders the access of a UT in the CON state). Probability $P_{\text {hin }}$ is related to the simultaneous occurrence of the following independent events.

- The UT which decides to access the shared channel on an idle slot is in a $\mathrm{HIN}_{i}$ state; this occurs with probability 
$P_{\text {useless }}$, given by

$$
P_{\text {useless }}=\left(\frac{N}{n} h\right) /\left(c+\frac{N}{n} h\right) .
$$

- Only one UT in the CON state attempts to transmit on the same idle slot. We derive the probability of this event, $P_{1 \mathrm{UT}}$, by conditioning on the number of contending UT's, $C$ : the conditioned probability is given by $C p(1-p)^{C-1}$. Hence, we use the distribution $\Theta_{C}(C)$ given in (35) to obtain $P_{1 \mathrm{UT}}$, as

$$
P_{1 \mathrm{UT}}=\sum_{C=0}^{M} C p(1-p)^{C-1} \Theta_{C}(C) .
$$

It follows that

$$
P_{\text {hin }}=P_{\text {useless }} P_{1 \mathrm{UT}} \text {. }
$$

Numerical evaluations of (42) have shown that the transmission attempts of UT's in hindering states slight influence the system performance. In particular, after solving the EPA system (19)-(22) for the parameter values given in Table I, $T_{f}=15 \mathrm{~ms}, n=3$, and $M=40 \mathrm{UT}$ 's/carrier (note that $N=21$ slots/frame), we have obtained $P_{\text {hin }} \approx$ $2 \%$. We have also verified that $P_{\text {hin }}$ is sufficiently small (i.e., below 3\%) if in the previous numerical example we consider $n=1$ and $T_{f}$ is increased up to $25 \mathrm{~ms}$.

\section{Theoretical AND Simulation Results}

This section deals with the validation of the analytical approach outlined in the previous sections. Numerical results have been obtained for the system parameter values shown in Table I. We have considered very long simulation runs in order to achieve an accurate and reliable estimation of $P_{\text {drop }}$. In particular, we have selected a duration of $50 \times 10^{6}$ slots which allows 5\% confidence interval for $P_{\text {drop }}$ for values of $M$ that guarantee $P_{\text {drop }} \geq 10^{-3}$ [27]; this is the range of $P_{\text {drop }}$ values which is useful for evaluating the capacity of both PRMA and PRMA-HS.

Fig. 10 shows simulation results for $P_{\text {drop }}$ for different values of $T_{f}$ and $p$, in the case of $M=34$ UT's/carrier and $n=1$. From Fig. 10 we can note that: 1) $P_{\text {drop }}$ increases when $p$ approaches zero or one for any $T_{f}$ value and 2) $P_{\text {drop }}$ significantly increases when $T_{f}$ is too low (e.g., $5 \mathrm{~ms}$ ) or becomes greater than $25 \mathrm{~ms}$. It is interesting to note that the best operating conditions for PRMA-HS are obtained when $T_{f}=15 \mathrm{~ms}$ and $p=0.4$, i.e., the optimized parameter values used in the case of the PRMA protocol (see Section III).

The behavior of parameter $\mu_{0.01}$ obtained from simulations for PRMA-HS is shown in Fig. 11 as a function of $T_{f}$ with $p=0.4$ and $n=1$. From these results we can state that the maximum value for $\mu_{0.01}$ (i.e., 1.63 conversations/channel) is achieved for $T_{f}=15 \mathrm{~ms}$. However, a good performance is also obtained for $T_{f}$ values within the range $12-25 \mathrm{~ms}$. By comparing Fig. 11 with Fig. 4, we can note that in the satellite case the efficiency of PRMA-HS is better than that of PRMA. Moreover, PRMA-HS has a better performance than PRMA also for high $T_{f} \approx \mathrm{RTD}_{\max }$ values. This is an interesting result which proves that the advantage of more transmission attempts

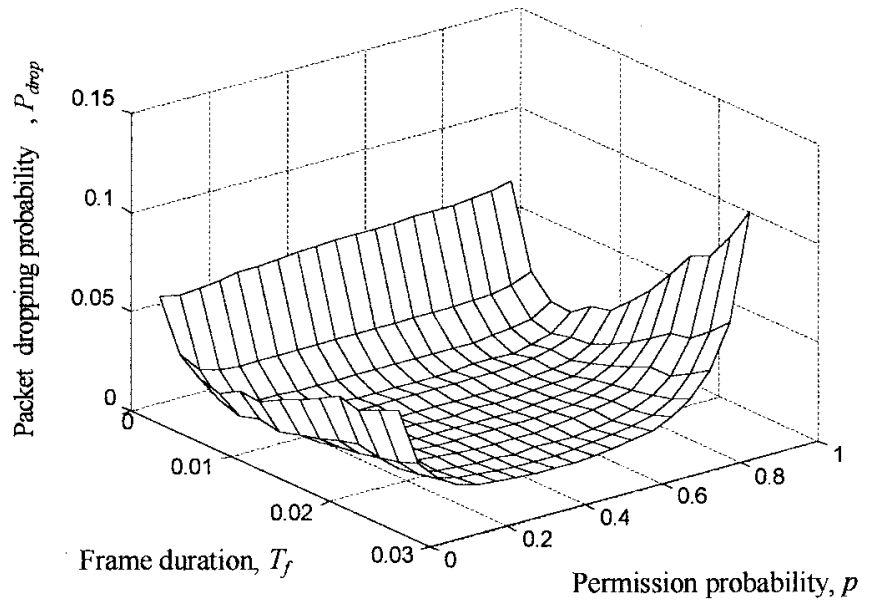

Fig. 10. Behavior of $P_{\text {drop }}$ as a function of $T_{f}$ and $p$ for $M=34$ UT's/carrier and $n=1$ (simulation results).

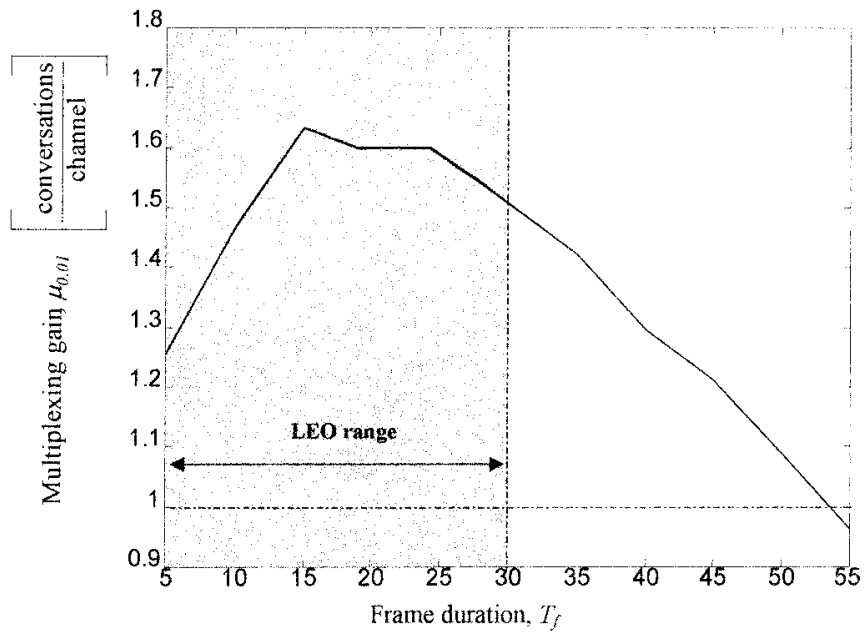

Fig. 11. The PRMA-HS multiplexing gain as a function of $T_{f}$ with $p=0.4$ and $n=1$ (satellite case with RTD $\max \approx T_{f}$ ).

within $D_{\max }$ overcomes the disadvantage due to transmission attempts from hindering states. Consequently, the PRMA-HS protocol maintains an acceptable performance also for higher altitude LEO satellite constellations than PRMA.

As proved by the following results (see Figs. 13 and 14), the PRMA-HS performance is quite insensitive to the value of $n$, for a given $T_{f}$ value. Therefore, the optimum values of both $T_{f}$ and $p$ for $n=1$ (i.e., $T_{f}=15 \mathrm{~ms}$ and $p=0.4$ ), can be considered as a good choice also for $n>1$.

Fig. 12 shows the behavior of $P_{\text {drop }}$ (theory and simulations) as a function of $M$ for the PRMA-HS technique with $p=0.4, T_{f}=15 \mathrm{~ms}$ (optimized values) and $\mathrm{RTD}_{\max }=5 \mathrm{~ms}$ (i.e., $n=3$ ). This graph shows that the proposed analytical approach efficiently predicts the system behavior around $P_{\text {drop }}=$ $1 \%$ (a similar behavior has been also obtained for other values of $p$ ). In particular, we note that both theory and simulations confirm that the capacity of a PRMA-HS carrier (i.e., $M_{0.01}$ ) is 39 UT's (hence, $\mu_{0.01}=1.63$ conversations $/$ channel).

Fig. 13 shows the comparison between the PRMA-HS protocol and the PRMA scheme in terms of $P_{\text {drop }}$ in LEO-MSS's 


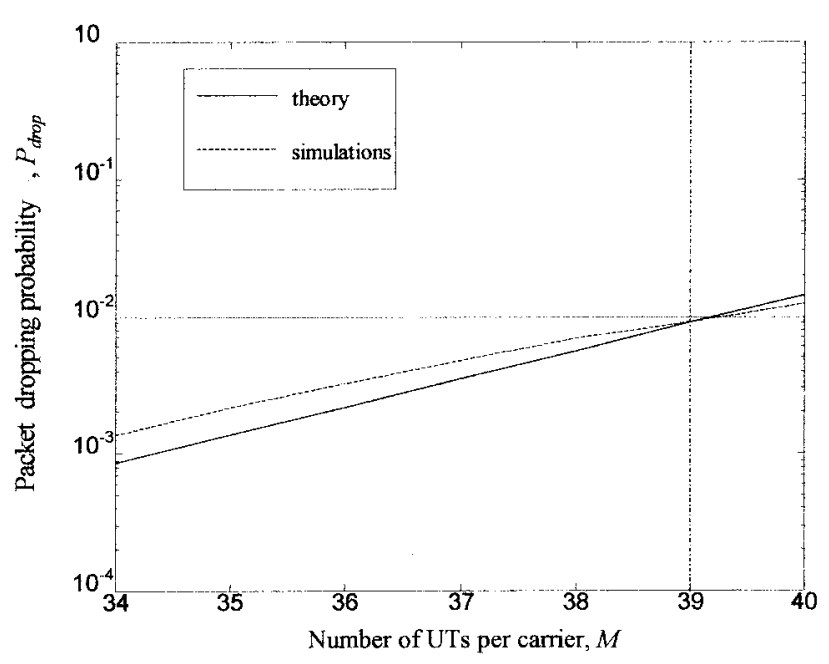

Fig. 12. Theoretical and simulation results for $P_{\text {drop }}$ versus $M$ for the proposed PRMA-HS scheme with $p=0.4, T_{f}=15 \mathrm{~ms}$, and $\mathrm{RTD}_{\max }=5$ ms.

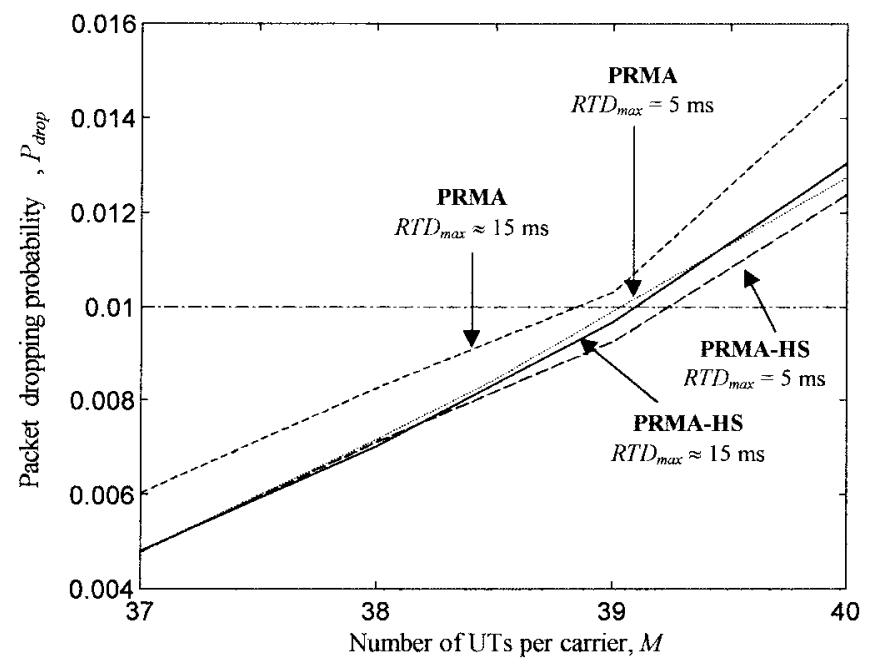

Fig. 13. Comparison in terms of $P_{\text {drop }}$ between the PRMA-HS protocol and the original PRMA scheme in LEO-MSS's with $p=0.4$ and $T_{f}=15 \mathrm{~ms}$, for both $n=3$ (i.e., $\mathrm{RTD}_{\max }=5 \mathrm{~ms}$ ) and $n=1$ (i.e., $\mathrm{RTD}_{\max } \approx 15 \mathrm{~ms}$ ).

for both $n=3$ (i.e., $\mathrm{RTD}_{\max }=5 \mathrm{~ms}$ ) and $n=1$ (i.e., $\mathrm{RTD}_{\max }=15 \mathrm{~ms}$ ) with $p=0.4$ and $T_{f}=15 \mathrm{~ms}$. We note that in this figure the performance difference between PRMA and PRMA-HS is slight for $n=3$ and significant for $n=1$. Hence, we may state that the performance of PRMA strongly depends on $n$ : an increase in RTD leads to a worse behavior. Whereas PRMA-HS is less sensitive to variations of RTD. This is an interesting result that makes this protocol quite insensitive to the variations of RTD experienced in LEO systems during call lifetime, mainly due to the motion of LEO satellites. Moreover, the PRMA-HS performance obtained for $n=1$ (i.e., $T_{f} \approx \mathrm{RTD}_{\max }$ ) can be considered as a conservative estimate of the PRMA-HS performance for any $\mathrm{RTD}_{\max }$ value less than $T_{f}$.

Fig. 14 shows the $P_{\text {drop }}$ behavior of PRMA-HS as a function of $M$ for both a terrestrial cellular system (where RTD $\approx$ 0 , and PRMA-HS is equivalent to PRMA) and LEO-MSS's,

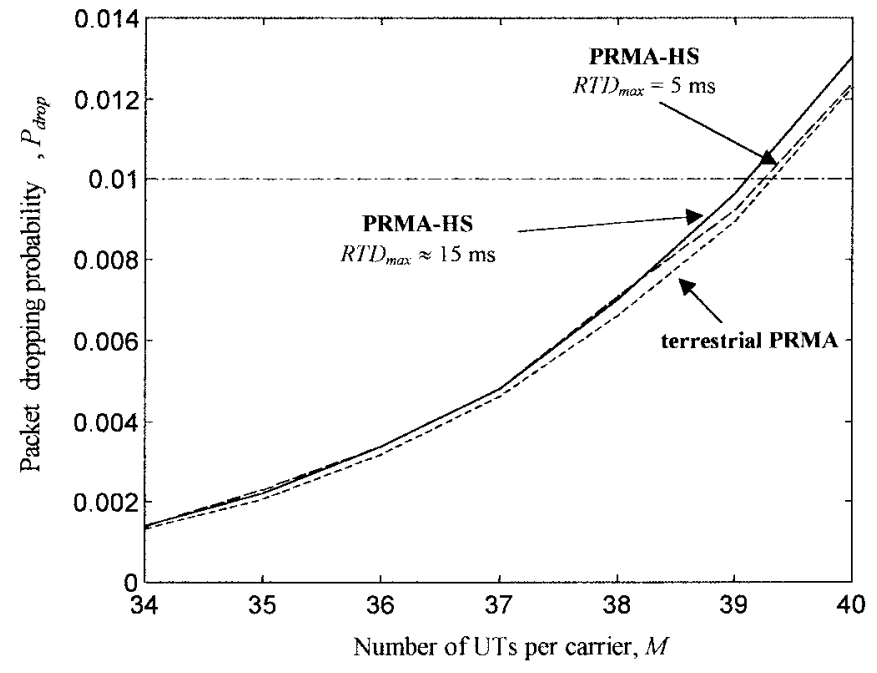

Fig. 14. Behavior of $P_{\text {drop }}$ for the PRMA-HS protocol in the LEO case (both $\mathrm{RTD}_{\max }=5 \mathrm{~ms}$ and $\mathrm{RTD}_{\max }=15 \mathrm{~ms}$ ) and the original PRMA scheme in the terrestrial case (RTD $=0)$, for $p=0.4$ and $T_{f}=15 \mathrm{~ms}$.

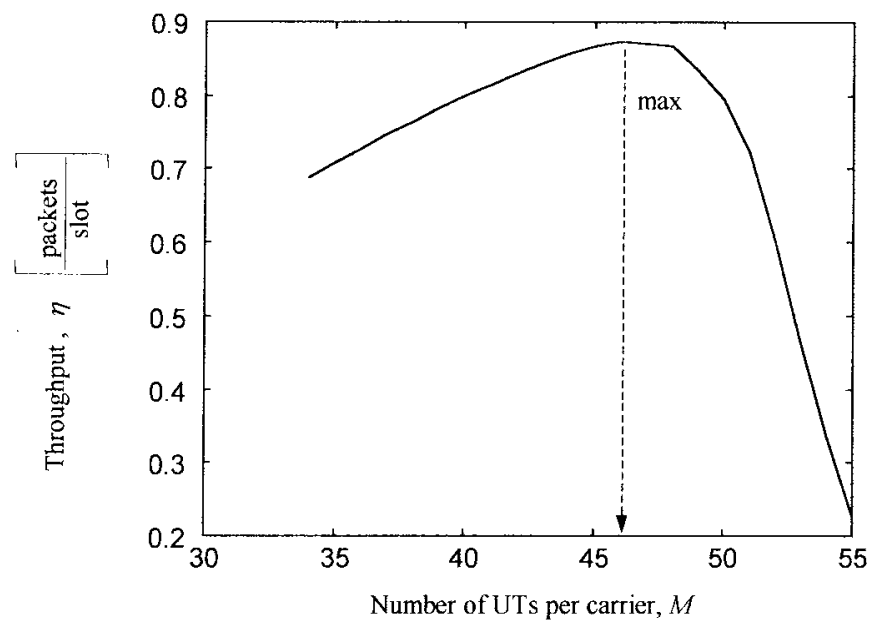

Fig. 15. System throughput (simulation results) for PRMA-HS as a function of the number of UT's per carrier, $M$, for $T_{f}=15 \mathrm{~ms}$, $\mathrm{RTD}_{\max }=5 \mathrm{~ms}$, and $p=0.4$.

by assuming $T_{f}=15 \mathrm{~ms}$ and $p=0.4$. We have considered two LEO-MSS's with RTD $\mathrm{RTax}_{\max }=5 \mathrm{~ms}$ and $\mathrm{RTD}_{\max }=15 \mathrm{~ms}$, respectively. From this graph we can note that the PRMA-HS protocol in the LEO case with $T_{f}=15 \mathrm{~ms}$ allows the same capacity of the PRMA protocol in the terrestrial environment (i.e., 39 UT's/carrier). This result represents a further proof that $T_{f}=15 \mathrm{~ms}$ and $p=0.4$ are optimum values for the PRMA-HS protocol in both terrestrial and LEO cellular systems, for the parameter values shown in Table I.

Fig. 15 shows the parameter $\eta$ for PRMA-HS as a function of $M$, for $T_{f}=15 \mathrm{~ms}, p=0.4$, and $n=3$. From this figure, we can note that $\eta$ has a maximum for $M$ about equal to 45 UT's/carrier. For $M$ values greater than 45 UT's/carrier, $\eta$ decreases. This behavior reveals a system congestion. For $M=M_{0.01}$ we obtain $\eta \approx 0.78$ packets/slot, as for the terrestrial PRMA [7]. Moreover, under the same conditions assumed for Fig. 15, Fig. 16 presents the comparison between analytical results and simulation ones for $\eta$ values in the region where $M$ is less than $F_{1} \approx 45.46$. [According to 


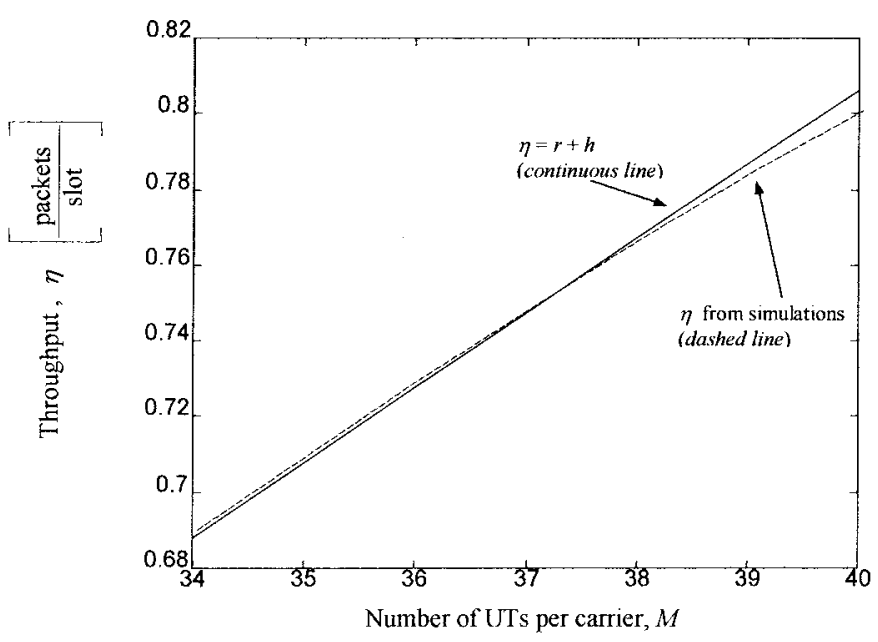

Fig. 16. System throughput (theory and simulation results) for PRMA-HS as a function of the number of UT's per carrier, $M$, (in the region where the EPA has a single solution) for $T_{f}=15 \mathrm{~ms}, \mathrm{RTD}_{\max }=5 \mathrm{~ms}$, and $p=0.4$.

Fig. 8, there is only one EPA solution and formula (39) can be used to evaluate theoretically $\eta$; since $\tilde{c}>c_{\min }$ for $p=0.4$, formula (39) cannot permit to show the maximum of $\eta$; see Section VI-B.] A good agreement is evident in this graph.

Finally, simulations have been carried out in order to highlight the better efficiency of PRMA-HS with respect to PRMA even in the presence of voice and data traffics. We have envisaged a data service with no special constraint on the end-to-end delay (e.g., file transfer or e-mail messages). Moreover, multimedia (data voice) UT's have been considered which may need to transmit (even contemporaneously) voice and data. The procedure used to acquire a reservation for data is the same as that considered for the transmission of talkspurts (see Section IV). If a UT already holds a reservation for the transmission of a talkspurt (data message) it must acquire another reservation for the transmission of an incoming data message (talkspurt). An exhaustive policy has been assumed for the transmission of data packets in the buffer of a UT. Data messages are generated by a UT according to a Poisson process. The message length in packets is geometrically distributed. We have assumed $T_{f}=15 \mathrm{~ms}$ and a permission probability equal to 0.4 for talkspurts. A lower permission probability (i.e., 0.2) has been considered for data messages in order to prioritize the (delay-sensitive) voice service.

Figs. 17 and 18, respectively, show $P_{\text {drop }}$ for voice packets and the mean message delay (in slots), $T_{\mathrm{msg}}$, for data messages as functions of the input data traffic $r_{d}$ (in packets/slot). In deriving these results we have considered 16 multimedia UT's per carrier with the system parameter values given in Table I and an average data message length equal to 20 packets. From Figs. 17 and 18 it is evident that the PRMA-HS protocol permits a higher value of $r_{d}$ without loosing the constraint $P_{\text {drop }} \leq 1 \%$ and a lower $T_{\text {msg }}$. Hence, we may conclude that PRMA-HS allows a better utilization of system resources than PRMA.

\section{CONCLUSIONS}

In this paper, we have carried out a feasibility study concerning the application of the PRMA protocol in mobile satellite

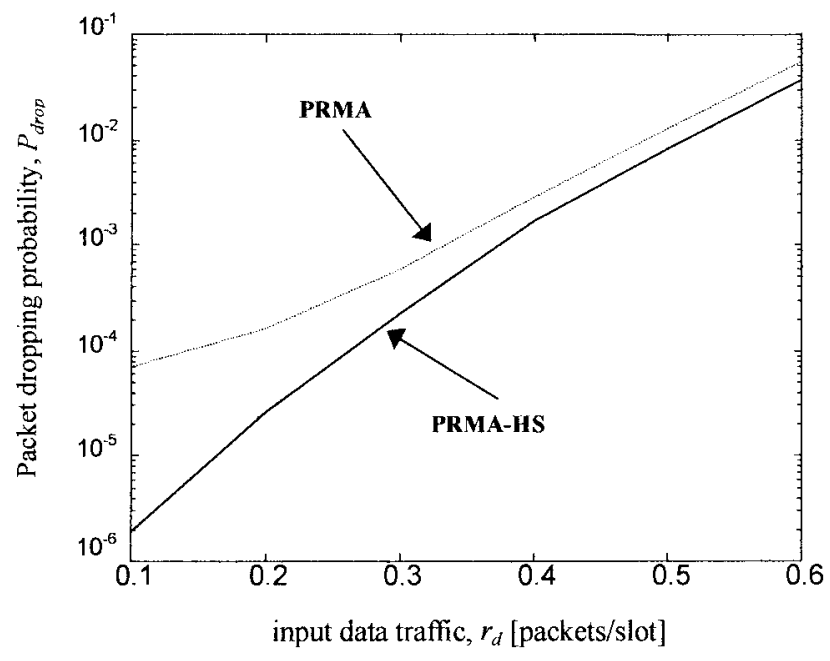

Fig. 17. Behavior of $P_{\text {drop }}$ for PRMA and PRMA-HS as a function of the input data traffic $r_{d}$, for $T_{f}=15 \mathrm{~ms}, n=1$, permission probability for voice $=0.4$, permission probability for data $=0.2,16$ multimedia UT's/carrier.

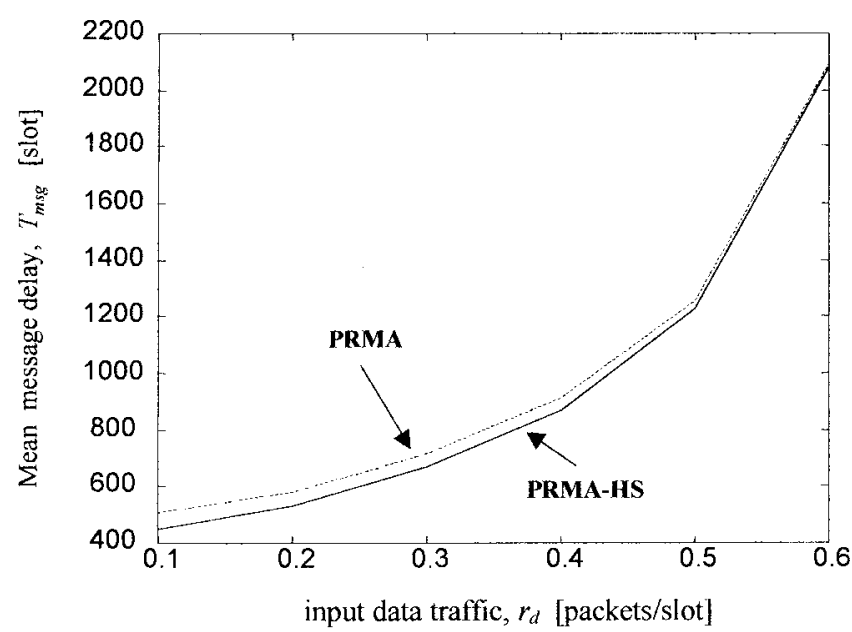

Fig. 18. Behavior of $T_{\mathrm{msg}}$ for PRMA and PRMA-HS as a function of the input data traffic $r_{d}$, for $T_{f}=15 \mathrm{~ms}, n=1$, permission probability for voice $=0.4$, permission probability for data $=0.2,16$ multimedia UT's/carrier.

systems where the round trip delay is not negligible with respect to the slot duration. We have shown that PRMA maintains a satisfactory performance in LEO systems, if the frame duration and the permission probability are suitably selected.

A novel protocol based on a modified version of the PRMA scheme, called PRMA with hindering states (PRMAHS), has been also proposed. The performance of the PRMAHS protocol has been evaluated by both an analytical approach and simulations. An important result shown here is that the PRMA-HS scheme in LEO systems achieves a performance equal to that of the PRMA protocol in terrestrial cellular systems. The better behavior of the PRMA-HS protocol with respect to the PRMA alternative has been also highlighted in the case of voice and data transmissions.

Finally, we can conclude that this paper has shown that the PRMA-HS protocol may be a good candidate as a unified 
MAC protocol for both the terrestrial and the satellite segment of the future UMTS.

\section{APPENDIX}

\section{THE NUMBER OF SOLUTIONS OF THE}

\section{EPA SYSTEM AND THEIR STABILITY}

In order to estimate the number of solutions of the EPA system [see (19) and (20)] for different values of $M$, we need to consider the maximum and the minimum of the curve $F(c, h)$, defined in (19), over the constraint $g(c, h)=0$ which is given in (20). We use the derivative of $F(c, h(c))$ with respect to $c$

$$
\frac{d F(c, h(c))}{d c}=\left[\frac{1}{\sigma}+\frac{N}{\gamma_{f}}\right] \frac{d h}{d c}+\left(1+\frac{\gamma}{\sigma}\right)
$$

where $d h / d c$ can be obtained through the rule for the derivation of the implicit function [28]: we compute the partial derivative of $g(c, h)$ with respect to $c$ and we equate this expression to zero; we obtain an equation that permits to derive the following implicit expression of $d h / d c$ :

$$
\frac{d h}{d c}=\frac{n(1-\gamma) p\left(\gamma_{f}-h\right) \xi}{(1-\gamma) p u c\left[n-\left(\gamma_{f}-h\right) N \ln (1-p)\right]+n \gamma_{f}}
$$

where

$$
\xi= \begin{cases}(1-p)^{(N / n) h}, & \text { for } c \leq 1 \\ (1-p)^{c+(N / n) h-1}[1+c \ln (1-p)], & \text { for } c>1\end{cases}
$$

and $h$ is a function of $c$ according to $g(c, h)=0$.

In order to obtain the maximum and the minimum of $F(c, h)$ over the constraint $g(c, h)=0$, we equate (A.1) to zero, where $d h / d c$ is given by (A.2) and (A.3). This method can be summarized as follows:

$$
\frac{d h}{d c}=-\frac{\left(1+\frac{\gamma}{\sigma}\right)}{\left[\frac{1}{\sigma}+\frac{N}{\gamma_{f}}\right]}
$$

where $d h / d c$ is given by (A.2) and (A.3).

The problem (A.4) has been solved through the Gauss-Newton iterative method [25]. Since multiple solutions are possible for (A.4), it is required to select the starting point sufficiently close to a solution for a fast convergence. This can be obtained by graphically identifying maximum and minimum points from Fig. 7 and by using these approximate solutions as starting points.

In order to establish if a solution of (A.4) represents a maximum or a minimum, we must study the disposition of $d h / d c$ as regards $-(1+\gamma / \sigma) /\left(1 / \sigma+N / \gamma_{f}\right)$. Let us consider the following example: the behaviors of both $h$ and $d h / d c$ as a function of $c$ have been shown in Fig. 19 for $N=21$ slots/carrier, $p=0.9$, and $n=3$. We may note that $d h / d c$ has a discontinuity for $c=1$ (due to the definition of $u$ ), which corresponds to the cusp point (and maximum) of $h$ as a function of $c$. In this graph the horizontal line represents the value $-(1+\gamma / \sigma) /\left(1 / \sigma+N / \gamma_{f}\right)$; the minimum and the maximum of $F(c, h)$ over the constraint can be graphically found by considering the intersections of $d h / d c$ with respect to

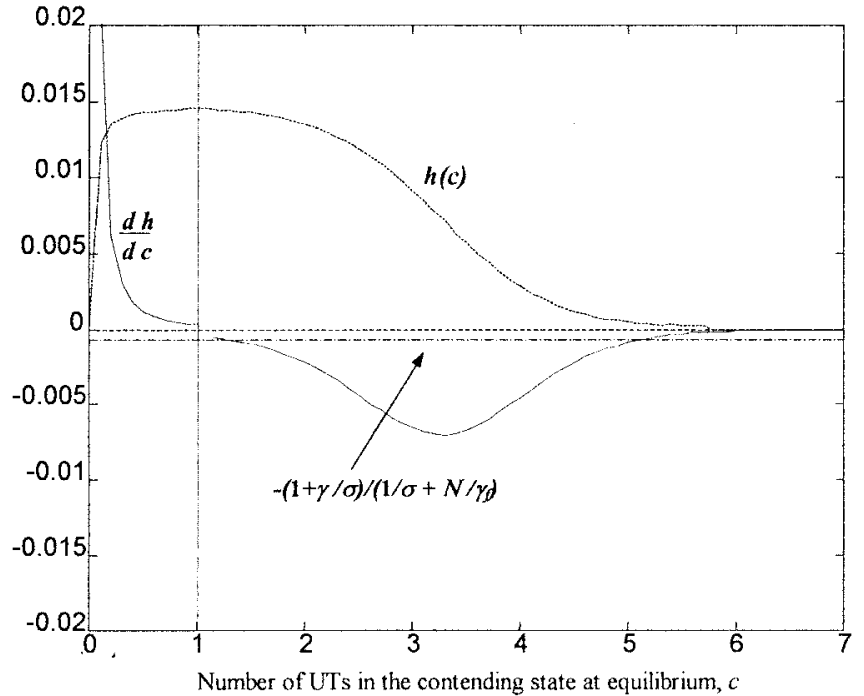

Fig. 19. Behavior of both $h$ and $d h / d c$ as a function of $c$ for $p=0.9$, $N=21$ slots/frame, $n=3$.

this line. In Fig. 19 there are two intersections. The intersection corresponding to the lower value of $c$ is related to a maximum, since $d h / d c$ passes from above to below the line $-(1+$ $\gamma / \sigma) /\left(1 / \sigma+N / \gamma_{f}\right)$ in this point. Whereas the intersection corresponding to the higher value of $c$ is related to a minimum. If $p$ decreases, these intersection points move toward higher values of $c$ and the minimum of $d h / d c$ approaches $0^{-}$[only $d h / d c$ depends on $p$, but the line $-(1+\gamma / \sigma) /\left(1 / \sigma+N / \gamma_{f}\right)$ does not]; we arrive at a value of $p$, here denoted by $p_{\min }=$ $p_{\min }(N, n, \sigma, \gamma)$, below which there is no intersection of $d h / d c$ with the line $-(1+\gamma / \sigma) /\left(1 / \sigma+N / \gamma_{f}\right)$. Through graphical evaluations, we have obtained that $p_{\min } \approx 0.205$ for $N=21$ slots/carrier and $n=3$. Then, for $p<p_{\min }$ we have no minimum and maximum for the $F(c, h)$ curve over the constraint $g(c, h)=0: F(c, h(c))$ has a monotonic behavior and the EPA system has a single solution for any value of $M$.

In order to study the stability of an equilibrium point for the PRMA-HS protocol we need to consider the outflow from the CON state which is given by $G(c, h)$ defined in (23). In particular, we must find maximum and minimum points of $G(c, h)$ as a function of $c$. The derivative of $G(c, h(c))$ with respect to $c$ is obtained as follows:

$$
\frac{d G(c, h(c))}{d c}=\frac{d h}{d c}+\gamma
$$

where $d h / d c$ is given by (A.2) and (A.3).

We are interested in investigating the sign of the derivative of $G(c, h(c))$ with respect to $c$; in particular, this derivative is equal to zero under the following condition:

$$
\frac{d h}{d c}=-\gamma
$$

where $d h / d c$ is given by (A.2) and (A.3).

A stable equilibrium point is characterized by a positive derivative of $G(c, h(c))$ with respect to $c$, where $h$ must fulfill $g(c, h)=0$ with $c \geq 0$ and $h \geq 0$. 
In (A.6), we must consider the intersection of $d h / d c$ with the line at the height $-\gamma$. This problem is similar to (A.4) where we study the intersection of $d h / d c$ with $-(1+\gamma / \sigma) /\left(1 / \sigma+N / \gamma_{f}\right)$. For the parameter values shown in Table I and $T_{f}=15 \mathrm{~ms}$, we have: $-\gamma \approx-7.14 \times 10^{-4}$ and $-(1+\gamma / \sigma) /\left(1 / \sigma+N / \gamma_{f}\right) \approx-7.119 \times 10^{-4}$ : these values are extremely close to each other and problems (A.4) and (A.6) have very similar solutions. Therefore, we can consider that the points for the maximum and the minimum of the function $F(c, h(c))$ are a very good approximation of the points for the maximum and the minimum of the function $\mathrm{P}\{G(c, h(c))$.

\section{REFERENCES}

[1] "Green paper on a common approach in the field of mobile and personal communications in the European Union," European Commission, 1994.

[2] H. Hansén, "Connection management functions of a private wireless ATM network," Master's thesis, Helsinki Univ. Technology, Mar. 13, 1996.

[3] E. Del Re, "A coordinated European effort for the definition of a satellite integrated environment for future mobile communications," IEEE Commun. Mag., pp. 98-104, Feb. 1996.

[4] "Mobile and personal communications," in Proceedings of the 2nd Joint COST 227/231 Workshop on Mobile Personal Communications, E. Del Re, Ed. Florence, Italy: Elsevier Science, 1995.

[5] D. J. Goodman, R. A. Valenzuela, K. T. Gayliard, and B. Ramanurthi, "Packet reservation multiple access for local wireless communications," IEEE Trans. Commun., vol. 37, pp. 885-890, Aug. 1989.

[6] D. J. Goodman, "Trends in cellular and cordless communications," IEEE Commun. Mag., pp. 31-40, June 1991.

[7] S. Nanda, D. J. Goodman, and U. Timor, "Performance of PRMA: A packet voice protocol for cellular systems," IEEE Trans. Veh. Technol., vol. 40, pp. 584-598, Aug. 1991.

[8] S. Nanda, "Stability evaluation and design of the PRMA joint voice data system," IEEE Trans. Commun., vol. 42, pp. 2092-2104, May 1994.

[9] SAINT Project (RACE II), Deliverable 11, "Radio resource management," R2117-FIU-DIE.DR.P211-B1, Sept. 1995.

[10] M. Frullone, G. Falciasecca, P. Grazioso, G. Riva, and A. M. Serra, "On the performance of packet reservation multiple access with fixed and dynamic channel allocation," IEEE Trans. Veh. Technol., vol. 42, pp. 78-86, Feb. 1993.

[11] W.-C. Wong, "Dynamic allocation of packet reservation multiple access carriers," IEEE Trans. Veh. Technol., vol. 42, pp. 385-392, Nov. 1993.

[12] Y. Li, S. Andersen, and B. Feng, "On the performance analysis of EPRMA protocol with Markov chain model," in Proc. GLOBECOM'95, Singapore, Nov. 13, 1995, pp. 1502-1506.

[13] G. Maral, J.-J. Ridder, B. G. Evans, and M. Richharia, "Low Earth orbit satellite systems for communications," Int. J. Sat. Commun., vol. 9, pp. 209-225, 1991.

[14] J. Restrepo and G. Maral, "Coverage concepts for satellite constellations providing communications services to fixed and mobile users," Space Commun., vol. 13, no. 2, pp. 145-157, 1995.

[15] _ "Constellation sizing for non-GEO 'Earth-fixed cell' satellite systems," in Proc. AIAA 16th Int. Communications Satellite Systems Conf. Exhibit, Washington, DC, Feb. 25-29, 1996, pp. 768-778.

[16] J. Gruber and L. Strawczynski, "Subjective effects of variable delay and speech clipping in dynamically managed voice systems," IEEE Trans. Commun., vol. COM-33, pp. 801-808, Aug. 1990.

[17] F. Ananasso and F. Delli Priscoli, "The role of satellites in personal communication services," IEEE J. Select. Areas Commun., vol. 13, pp. 180-196, Feb. 1995

[18] R. V. Cox, "Three new speech coders from the ITU cover a range of applications," IEEE Commun. Mag., vol. 35, pp. 40-47, Sept. 1997.

[19] J. F. Hayes, Modeling and Analysis of Computer Communication Networks. New York: Plenum, 1986.

[20] L. Kleinrock, Queuing Systems. New York: Wiley, 1976.

[21] A. Fukuda and S. Tasaka, "The equilibrium point analysis-A unified analytic tool for packet broadcast networks," in Proc. IEEE GLOBECOM'83, San Diego, CA, Nov. 1983, pp. 33.4.1-33.4.8.
[22] S. Tasaka, "Performance comparison of multiple access protocols for satellite broadcast channels," in Proc. IEEE GLOBECOM'83, San Diego, CA, Nov. 1983, pp. 35.3.1-35.3.8.

[23] G. Wu, "Analysis of an integrated voice data transmission system using PRMA,” IEEE Trans. Veh. Technol., vol. 43, pp. 289-297, May 1994.

[24] X.-F. Dong and L.-M. Li, "A spread spectrum PRMA protocol with randomized arrival times for microcellular systems," in Proc. GLOBECOM'96, London, U.K., Nov. 18-22, 1996.

[25] Matlab-Reference Guide, The Math Works, Inc., Natick, MA, Aug. 1992.

[26] H. Kobayashi and A. G. Konheim, "Queuing models for computer communications system analysis," IEEE Trans. Commun., vol. COM-25, pp. 2-28, Jan. 1977.

[27] D. D. Dimitrijevic and J. Vucetic, "Design and performance analysis of the algorithms for channel allocation in cellular networks," IEEE Trans. Veh. Technol., vol. 42, pp. 526-534, Nov. 1993.

[28] M. Krasnow, A. Kiselev, G. Makarenko, and E. Shikin, Mathematical Analysis for Engineers. Moscow, Russia: MIR, 1990.

Enrico Del Re (M'78-SM'84) was born in Florence, Italy, in 1947. He received the Dr.Ing. degree in electronics engineering from the University of Pisa, Pisa, Italy, in 1971.

Until 1975, he worked in public administration and private firms, where he was involved in the analysis and design of the telecommunication and air traffic control equipment and space systems. Since 1975, he has been with the Department of Electronics Engineering, University of Florence, Florence, Italy, first as a Research Assistant and then as an Associate Professor. Since 1986, he has been a Professor at the University of Florence. During the academic year 1987-1988, he was on leave from the University of Florence for a nine-month period of research at the European Space Research and Technology Center of the European Space Agency, The Netherlands. $\mathrm{He}$ is the Head of the Digitial Signal Processing and Telematics Laboratory (LENST), Department of Electronic Engineering, University of Florence. $\mathrm{He}$ also teaches a course in digital signal processing. His main research interests are digital signal processing, digital transmission techniques, and communication networks, on which he has published more than 150 papers in international journals and conferences. He is the coeditor of Satellite Integrated Communications Networks (North-Holland, 1988) and a coauthor of Data Compression and Error Control Techniques with Applications (New York: Academic, 1985).

Dr. Del Re has been the Chairman of the European Project COST 227 "Integrated space/terrestrial mobile networks" and is now Chairman of the COST 252 Project "Evolution of satellite personal communications from second to future generation systems." He received the 1988/89 premium from the IEE (U.K.) for the paper "Multicarrier demodulator for digital satellite communication systems." He has been included in Who's Who in the World. $\mathrm{He}$ is a member of the AEI and European Association for Signal Processing (EURASIP).

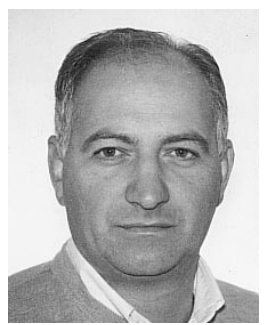

Romano Fantacci (S'82-M'87-SM'91) was born in Pistoia, Italy. He received the Dr.Ing. degree in electronics engineering in 1982 and the Ph.D. degree in telecommunication engineering in 1987, both from the University of Florence, Florence, Italy.

In 1982, he joined the Electronics Engineering Department, University of Florence, as a Researcher in Computer Communications, where he is now an Associate Professor of Telecommunication Networks. During the first stage of his research activity, he worked on satellite communication systems. In particular, he has been involved in several European Space Agency (ESA) and INTELSAT advanced research projects. His present research interests involve digital communications, computer communications, queueing theory, fast packet switching, and mobile communication networks. He is the author of works that have been published in several journals in communication science.

Dr. Fantacci has been involved in several European (RACE) and nationwide research projects sponsored by the Italian National Council of Research (C.N.R.) and Italian Minister of Education and Research (MURST). 


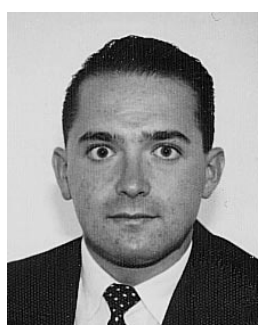

Giovanni Giambene (S'94-M'97) received the Dr.Ing. degree in electronics in 1993 and the Ph.D. degree in telecommunications and informatics in 1997, both from the University of Florence, Florence, Italy.

Since 1994, he has been with the Electronic Engineering Department, University of Florence, as a Research Assistant. His research interests include mobile cellular communication networks, personal communication services, channel allocation techniques, and queuing theory.

Dr. Giambene was the Technical External Secretary of the European Community Project COST 227 "Integrated space/terrestrial mobile networks." He also contributed to the "resource management" activity of Working Group 3000 within the RACE Project called "Satellite integration in the future mobile network" (SAINT, RACE 2117).

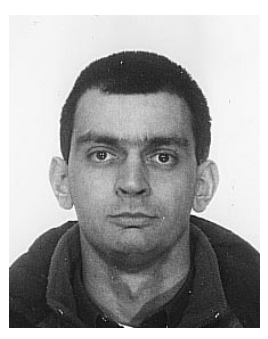

Walter Sergio was born in Trieste, Italy, in 1970. He received the B.S. degree (Laurea) from the University of Florence, Italy, in 1997.

$\mathrm{He}$ is a Research Assistant in the Electronic Department of the University of Florence. His main interests are mobile communications, satellite communications, and wireless links. 